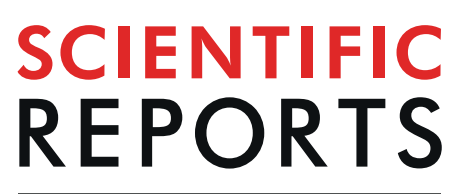

\title{
CETSA-based target engagement of taxanes as biomarkers for efficacy and resistance
}

\author{
Anette Langebäck ${ }^{1,6}$, Smaranda Bacanu ${ }^{1,6}$, Henriette Laursen ${ }^{1,6}$, Lisanne Moutt, \\ Takahiro Seki ${ }^{5}$, Sigrun Erkens-Schulze ${ }^{4}$, Anderson Daniel Ramos ${ }^{1}$, Anna Berggren ${ }^{1}$, Yihai Cao ${ }^{5}$, \\ Johan Hartman $\mathbb{1}^{1}{ }^{1}$, Wytske van Weerden ${ }^{4}$, Jonas Bergh ${ }^{1}$, Pär Nordlund ${ }^{1,2,3^{*}}$ \& Sara Lööf ${ }^{1}$
}

The use of taxanes has for decades been crucial for treatment of several cancers. A major limitation of these therapies is inherent or acquired drug resistance. A key to improved outcome of taxane-based therapies is to develop tools to predict and monitor drug efficacy and resistance in the clinical setting allowing for treatment and dose stratification for individual patients. To assess treatment efficacy up to the level of drug target engagement, we have established several formats of tubulin-specific Cellular Thermal Shift Assays (CETSAs). This technique was evaluated in breast and prostate cancer models and in a cohort of breast cancer patients. Here we show that taxanes induce significant CETSA shifts in cell lines as well as in animal models including patient-derived xenograft (PDX) models. Furthermore, isothermal dose response CETSA measurements allowed for drugs to be rapidly ranked according to their reported potency. Using multidrug resistant cancer cell lines and taxane-resistant PDX models we demonstrate that CETSA can identify taxane resistance up to the level of target engagement. An imaging-based CETSA format was also established, which in principle allows for taxane target engagement to be accessed in specific cell types in complex cell mixtures. Using a highly sensitive implementation of CETSA, we measured target engagement in fine needle aspirates from breast cancer patients, revealing a range of different sensitivities. Together, our data support that CETSA is a robust tool for assessing taxane target engagement in preclinical models and clinical material and therefore should be evaluated as a prognostic tool during taxane-based therapies.

The use of taxanes has been a cornerstone in the treatment of cancers for several decades. Today taxanes are used in several cancer types and are a part of first line treatment in breast, non-small-cell lung cancer, castration-resistant prostate cancer, esophageal, head and neck cancers, and Kaposi sarcoma ${ }^{1-3}$.

Taxanes are particularly important in the treatment of both early-stage and metastatic breast cancer patients where robust improvements of overall survival have been shown ${ }^{4,5}$. Both paclitaxel, a substance isolated from the Pacific yew tree (Taxus brevifolia), and docetaxel, a more potent semisynthetic derivate of paclitaxel, derived from the European yew tree (Taxus baccata), are standard treatment in breast cancer. Taxanes are also used for prostate cancer patients and are currently the only type of chemotherapy used for this type of cancer. Although the mainstay therapy for metastatic prostate cancer has been hormone depletion, no effective therapy was available for patients who had progressed under androgen deprivation therapy until the introduction of docetaxel in $2004^{6}$. Currently the treatment landscape for patients with castrate-resistant prostate cancer (CRPC) has vastly expanded, including cabazitaxel. This novel taxane has been shown to remain effective in docetaxel-resistant CRPC patients ${ }^{7}$. Of note, the addition of docetaxel in parallel to androgen deprivation therapy was recently shown to increase overall survival, suggesting that an earlier switch to taxane regime might be favourable in these cases ${ }^{8}$.

The taxanes exert their effect by binding to $\beta$-tubulin on the luminal side of microtubules leading to the stabilization of microtubules via inhibition of depolymerization ${ }^{9}$. Therefore taxanes suppress microtubule dynamics,

\footnotetext{
${ }^{1}$ Department of Oncology-Pathology, Karolinska Institutet, BioClinicum, Solna, 171 64, Sweden. ${ }^{2}$ School of Biological Sciences, Nanyang Technological University, Singapore 637551, Singapore. ${ }^{3}$ Institute of Molecular and Cell Biology, A*STAR, Singapore 138673, Singapore. ${ }^{4}$ Department of Urology, Erasmus Medical Centre, Rotterdam, The Netherlands. ${ }^{5}$ Department of Microbiology, Tumor and Cell Biology, Karolinska Institutet, Solna, 171 65, Sweden. ${ }^{6}$ These authors contributed equally: Anette Langebäck, Smaranda Bacanu, Henriette Laursen and Lisanne Mout. *email: par.nordlund@ki.se
} 
with the major mechanism for cell toxicity being the effect on the mitotic spindle, which leads to cell cycle arrest typically in G2- and M-phase, followed by cell death in rapidly dividing cells ${ }^{10}$. Vinca alkaloids is another family of tubulin modulators, including vinorelbine and vincristine, which act by inhibiting microtubule polymerization, also arresting cells in G2- and M-phase.

Taxanes are widely used for both early- and late-stage disease, but as for other cytotoxic cancer drugs, inherent or acquired resistance is often seen, leading to lack of efficacy or time-limited responses, and eventually progressive disease and mortality. There have been multiple mechanisms attributed to taxane resistance where a specific focus has been on the overexpression of efflux pumps, for example P-glycoprotein 1 (MDR1) or down-regulation of influx transporters ${ }^{11}$. The activation of efflux pumps leads to depletion of the intracellular pool of the taxane, which affects the extent of binding to $\beta$-tubulin, i.e. decrease the target engagement (TE). Attenuated TE has also been suggested to occur due to point mutations or post translational modifications of $\beta$-tubulin, modified expression levels of microtubule-associated proteins (MAPs) or altered levels of tubulin isoforms such as up-regulation of TUBB3 ${ }^{12-14}$. However, the mechanisms for how TUBB3 overexpression overcomes cell cycle arrest remains only partially defined. Other general mechanisms for resistance to cytotoxic drugs have also been suggested to affect taxane sensitivity such as altered expression of anti-apoptotic proteins (e.g. Bcl-2 and Bcl-xL) ${ }^{15,16}$ and NFkB modulation ${ }^{17}$.

However, although several potential mechanisms and biomarkers for cellular drug efficacy and resistance have been proposed, none of these have been proven predictive for efficacy in the clinical setting. This is unfortunate since different taxanes appear to have different efficacy profiles, as well as different adverse effects, and thus biomarkers and predictive assays could potentially help in stratifying drug or drug combinations at different stages of taxane-based combination therapy.

A basic requirement for taxane efficacy is sufficient TE of $\beta$-tubulin. After the drug has reached the tumor environment, TE is mainly determined by cellular influx, efflux, and drug catabolism, yielding the effective intracellular drug concentration. During therapy the pharmacokinetic (PK) profile in the individual patient will also affect TE by determining the amount of taxanes reaching the tumour environment. To optimize PK, drug monitoring is sometimes used during taxane therapy to demonstrate appropriate serum concentrations of the drug ${ }^{18}$.

Considering the critical role of TE for drug efficacy, information on absolute and relative TE for different drugs in patient-derived samples could potentially help in stratification of cancer therapies with taxanes. However, direct measurements of TE in cells and tissues have previously been very challenging when no general technique for such measurements has been available. To meet this challenge, our lab has introduced the Cellular Thermal Shift Assay (CETSA), a biophysical assay which allows for TE to be measured in intact cells and tissues ${ }^{19}$. CETSA can directly assess drug binding at the target protein level (the protein reports) by applying the critical heating step while cells are still intact and the target protein is in its proper cellular environment. After determining overall melting behavior of a target protein, isothermal dose response (ITDR) curves can be determined with CETSA to assess relative doses needed to obtain TE. Combined with mass spectrometry (MS-CETSA) the method has allowed for comprehensive characterization of both direct drug binding, and protein interaction state changes induced by downstream and stress effects introduced by drugs.

Although CETSA is most often explored to study TE in cell cultures, single protein CETSA experiments on tissue samples have previously been done for drug treated mice, for example, in the initial CETSA study for METAP $2^{19}$ and in several mice tissues for RIPK $1^{20}$. Proteome wide MS-CETSA experiments have also recently been performed in multiple tissues of drug treated mice (ref. https://www.biorxiv.org/content/10.1101/500306v1).

In the present work we implemented different formats of tubulin-specific CETSA and used these to study taxane TE in cell lines of breast and prostate cancer as well as mouse xenografts. Using cognate pairs of sensitive and resistant cell lines we show that CETSA can efficiently reveal the presence of drug resistance mechanisms. Finally, using miniaturized CETSA we demonstrate that TE responses can be measured in fine needle aspirates from breast cancer patients and therefore can be used to validate tubulin CETSA as a potential prognostic biomarker for taxane sensitivity in clinical trials.

\section{Results}

Tubulin melting behavior and drug stabilization in western blot-based CETSA. To validate the applicability of CETSA to assess TE for tubulin-targeted drugs we implemented several CETSA formats as shown in Fig. 1A. We first developed a western blot-based assay to investigate the feasibility of tubulin-based CETSA. Initially melt curves for $\alpha$ - and $\beta$-tubulin were generated in K562-cells, a suspension cell line originating from myelogenous leukemia. Both $\alpha$ - and $\beta$-tubulins had relatively high melting temperatures, around $61-63^{\circ} \mathrm{C}$, compared to $52^{\circ} \mathrm{C}$ as the average melting temperature of proteins of the human proteome $e^{21}$. The high melting temperatures of tubulins might be due to stabilizing effects of the extensive protein-protein interactions made within the microtubule polymer. This is consistent with the finding that the non-polymerized subunits of tubulin, in lysates, melt more than $10^{\circ} \mathrm{C}$ earlier (Fig. S1A,B).

In addition to paclitaxel, which preferentially binds to a site on $\beta$-tubulin localized in the lumen of microtubules leading to stabilization of microtubules, we used vinorelbine, that binds to the vinca-binding domain, a site on $\beta$-tubulin distinct from the taxane-binding domain and which inhibits tubulin polymerization ${ }^{22-24}$. Treatment of K562-cells for $1 \mathrm{~h}$ with both drugs produced a significant stabilization of $\beta$-tubulin with the largest difference between vehicle and treated seen around $63^{\circ} \mathrm{C}$ (Fig. $1 \mathrm{~B}$ and Table 1). A significant shift was also observed for $\alpha$-tubulin following treatment with paclitaxel (Fig. 1C and Table 1). However, no shifts were observed in cell lysates (Fig. S1A,B), consistent with the fact that drug binding is considered to occur only with intact microtubules ${ }^{12}$. In lysate, microtubules are depolymerized and tubulin is only present as soluble dimers. Although the drugs used bind specifically to the $\beta$-tubulin subunit, both the $\alpha$ - and $\beta$-subunits show shifts in CETSA melt curves (Fig. 1B-E and Table 1). We recently described the correlation of CETSA melt curves of interacting proteins and named this phenomena thermal proximity co-aggregation (TPCA $)^{25,26}$, which is the likely explanation for the correlated shifts 
A
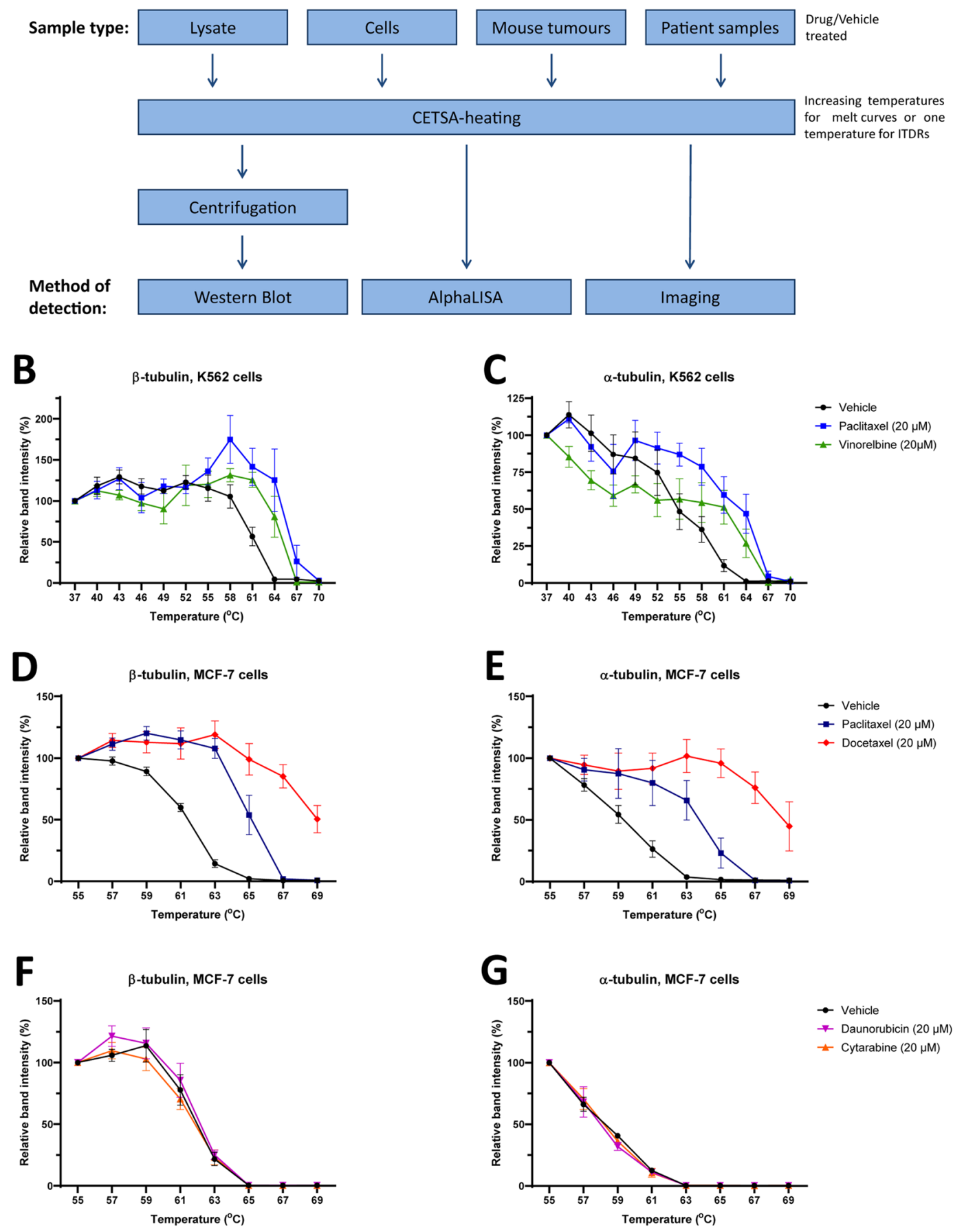

Figure 1. CETSA shows distinct melt curves for $\alpha$ - and $\beta$-tubulin that shift upon drug binding in cancer cells. Schematic overview of the CETSA method (A). Western blot-CETSA shows that the tubulin-binding drugs paclitaxel and vinorelbine $(20 \mu \mathrm{M})$ produce clear shifts for $\beta$-tubulin in K562-cells (B and C). In MCF-7 cells, the two taxanes, paclitaxel and docetaxel $(20 \mu \mathrm{M})$, produce significant CETSA shifts for both $\beta$-tubulin and $\alpha$-tubulin (D and $\mathbf{E}$ ). Daunorubicin and cytarabine (negative controls) produced no shift in both $\beta$-tubulin and $\alpha$-tubulin $(\mathbf{F}$ and $\mathbf{G})$. All data represent the mean \pm S.E.M from independent experiments $(n=5-6$ in $\mathbf{D}$ and $\mathbf{E}$, and $\mathbf{n}=3$ in $\mathbf{B}, \mathbf{C}, \mathbf{F}$ and $\mathbf{G}$ ) and are presented as a percentage of the signal detected at the lowest temperature in each melt curve.

of the tubulin subunits. CETSA melt curves were also generated in MCF-7 cells, a commonly used model system for hormone-positive breast cancer. Paclitaxel generated clear shifts in both $\alpha$ - and $\beta$-tubulin in these cells, while docetaxel produced even larger shifts (Fig. 1D,E and Table 1). As negative controls we have generated melt curves for MCF-7 cells treated with the anthracycline daunorubicin, or with the pyrimidine analogue cytarabine which, 


\begin{tabular}{|c|c|c|c|c|c|c|}
\hline \multirow[b]{2}{*}{ K562 cells } & \multicolumn{3}{|l|}{$\beta$-tubulin } & \multicolumn{3}{|l|}{$\alpha$-tubulin } \\
\hline & $\mathrm{T}_{\mathrm{m}}\left({ }^{\circ} \mathrm{C}\right)$ & $\Delta \mathrm{T}_{\mathrm{m}}\left({ }^{\circ} \mathrm{C}\right)$ & Significance* & $\mathrm{T}_{\mathrm{m}}\left({ }^{\circ} \mathrm{C}\right)$ & $\Delta \mathrm{T}_{\mathrm{m}}\left({ }^{\circ} \mathrm{C}\right)$ & Significance* \\
\hline Vehicle & $61,0 \pm 0,6$ & - & - & $54,0 \pm 2,1$ & - & - \\
\hline Paclitaxel $(20 \mu \mathrm{M})$ & $66,1 \pm 0,8$ & $5,1 \pm 1,0$ & $* * *$ & $63,0 \pm 1,0$ & $9,0 \pm 3,1$ & $*$ \\
\hline Vinorelbine $(20 \mu \mathrm{M})$ & $64,7 \pm 0,8$ & $3,7 \pm 1,1$ & $*$ & $62,5 \pm 0,5$ & $8,5 \pm 3,8$ & ns \\
\hline MCF-7 cells & $\mathrm{T}_{\mathrm{m}}\left({ }^{\circ} \mathrm{C}\right)$ & $\Delta \mathrm{T}_{\mathrm{m}}\left({ }^{\circ} \mathrm{C}\right)$ & Significance* & $\mathrm{T}_{\mathrm{m}}\left({ }^{\circ} \mathrm{C}\right)$ & $\Delta \mathrm{T}_{\mathrm{m}}\left({ }^{\circ} \mathrm{C}\right)$ & Significance* \\
\hline Vehicle & $61,2 \pm 0,2$ & - & - & $58,9 \pm 0,3$ & - & - \\
\hline Paclitaxel $(20 \mu \mathrm{M})$ & $64,9 \pm 0,2$ & $3,7 \pm 0,3$ & ***** & $63,8 \pm 0,7$ & $4,9 \pm 0,6$ & *** \\
\hline Docetaxel $(20 \mu \mathrm{M})$ & $68,9 \pm 0,4$ & $7,7 \pm 0,4$ & $* * * *$ & $68,5 \pm 0,6$ & $9,6 \pm 0,6$ & $* * * *$ \\
\hline Daunorubicin $(20 \mu \mathrm{M})$ & $61,9 \pm 0,3$ & $0,7 \pm 0,4$ & $\mathrm{~ns}$ & $57,7 \pm 0,3$ & $-1,2 \pm 0,6$ & ns \\
\hline Cytarabine $(20 \mu \mathrm{M})$ & $61,7 \pm 0,3$ & $-0,5 \pm 0,4$ & ns & $58,1 \pm 0,3$ & $-0,7 \pm 0,6$ & ns \\
\hline
\end{tabular}

Table 1. Overview of melting temperatures $\left(\mathrm{T}_{\mathrm{m}}\right)$, CETSA shifts $\left(\Delta \mathrm{T}_{\mathrm{m}}\right)$, with associated standard errors and statistical significance of the measured CETSA shift for the data presented in Fig. 1B-G. *The significance of the CETSA shifts was calculated using one-way ANOVA for Tm values from independent experiments. Adjusted $\mathrm{P}$-values ns $\mathrm{P}>0.05, * \mathrm{P}<0.05, * * \mathrm{P}<0.01, * * * \mathrm{P}<0,001, * * * * \mathrm{P}<0,0001$ compared to vehicle.

as expected, produced no shifts in neither $\alpha$-nor $\beta$-tubulin (Fig. 1F,G). Since the temperatures needed to generate tubulin melting are high, there might be a risk of losing cell membrane integrity during heating. We therefore tested the temperature and time dependent effects on the cell membranes in K562 and MCF-7 cells using a trypan blue assay (Fig. S1C-F). Although some cell lysis was induced above $60^{\circ} \mathrm{C}$, the majority of cells remained intact up to $63^{\circ} \mathrm{C}$. In a time dependent experiment, the cell lysis occurred mainly after 1 minute of the total 3 minutes heating time (Fig. S1E,F), which is beyond the time point when the most prominent effects on protein stability are expected (the remaining heating time is expected to mainly drive the efficient precipitation of unfolded protein).

Importantly, when the melting temperature of tubulin in lysates is much lower than in cells, proteins from lysed cells will not be seen above $60^{\circ} \mathrm{C}$. Furthermore, no stabilizing effect by taxanes can be observed on tubulin in lysed cells when tubulin is then present only as soluble dimers, as discussed above. Therefore, the heat-induced lysis of a fraction of the cells is not likely to quantitatively affect the TE measurements with tubulin CETSA.

Miniaturization of CETSA for $\beta$-tubulin using AlphaLISA. Since measurements on clinical samples typically are limited by the amount of available cells, and screening studies using cell lines can require high sample throughput, we established a miniaturized tubulin CETSA based on AlphaLISA (overview of method in Fig. 2A). Antibody pairs can be chosen to only detect native protein in these assays with the advantage that, after heating, centrifugation of the lysate is not needed to remove aggregated protein (Figs. 2C,D and S2B,C). Several combinations of antibodies were tested and two pairs were found to give a robust signal: pair 2 consisting of ab6046 (Abcam) in combination with sc-398937 (Santa Cruz) and pair 8 consisting of ab6046 (Abcam) and T5201 (Sigma). Both pairs could detect CETSA melt curves and target stabilization in response to docetaxel and vinorelbine with very similar shape as detected with western blot (Fig. 2C,D, Table 2, and Fig. S2B,C). Also CETSA ITDR experiments performed at $64^{\circ} \mathrm{C}$ showed very similar curves with both detection methods (Fig. S2A).

In order to optimize the cell numbers required for these assays different amounts of lysate were analyzed with both antibody pairs (Figs. 2E and S2D). The two pairs showed similar curves with pair 8 being somewhat more sensitive and having slightly shorter linear range. To increase the sensitivity even further, the pair 8 antibodies were directly conjugated to the AlphaLISA beads (Figs. 2B and S2E). Also a polyclonal SOD-1 antibody (R\&D systems AF3418) was used in a conjugated AlphaLISA assay to function as loading control since SOD-1 is stable also at higher temperatures ${ }^{27}$ (Fig. S2G). The SOD1 assay had a linear range up to 500 cells (Fig. S2H). Interestingly, a time course experiment showed the stabilization of tubulin by docetaxel in K562 cells to be fully saturated already after 5 min drug treatment (Fig. S2F).

Detection of acquired drug resistance at the TE level using CETSA. To investigate the feasibility of using CETSA for assessing acquired drug resistance, multidrug-resistant K562-R cells were studied. The K562-R cells are previously described as vincristine-resistant due to overexpression of $\mathrm{P}-\mathrm{gp}^{28}$. We used AlphaLISA as readout method for ITDR-CETSA in order to compare the dose dependence of different taxanes in K562-R and the parental counterpart K562-P after a $1 \mathrm{~h} 45 \mathrm{~min}$ exposure to drugs. The ITDR-CETSA data showed that $\mathrm{K} 562-\mathrm{R}$ cells required in the range of 5,3x higher paclitaxel or docetaxel concentrations to establish the same TE response as in the parental cells (Fig. 3A,E, and Table 2) despite no difference in the total amount of tubulin (Fig. 3K). Measurements of cell viability after $72 \mathrm{~h}$ using the resazurin viability assay confirmed resistance in the K562-R cells compared to K562-P (Fig. 3B,F, and Table 2). Interestingly, the differences in both cell survival and CETSA shifts were abolished by the potent P-gp-inhibitor tariquidar (Fig. 3C,D,G,H, and Table 2), directly supporting that the resistance mechanism is indeed due to up-regulation of P-glycoprotein. Furthermore, these cells were exposed to epothilone B, a tubulin targeting drug which is not a P-glycoprotein substrate. Epothilone B induced almost identical CETSA curves in K562-R as in K562-P and did not show any difference in the resazurin assay, further supporting that the observed difference between the two cells lines is mainly P-gp-dependent. Consistent with these findings, the protein levels of P-gp were found to be high in K562-R and barely detectable in K562-P as examined by western blot (Fig. 3L). Together these data illustrate that CETSA can be used to detect development of acquired drug resistance, as well as report on the mechanism of resistance in specific cases. 
A

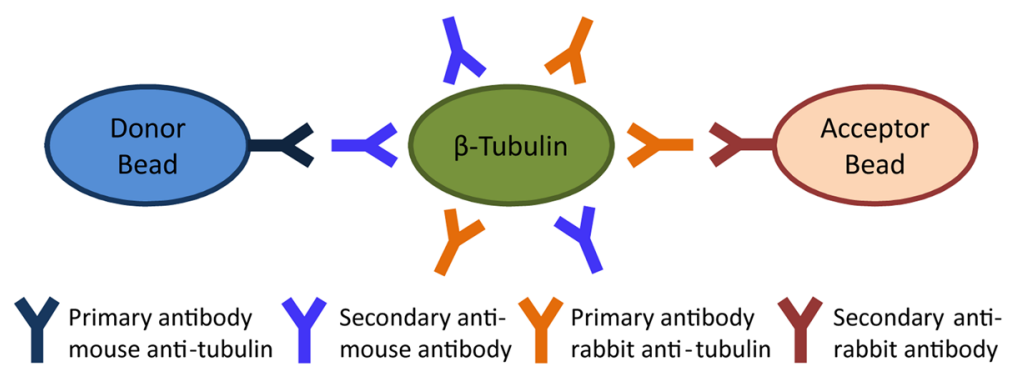

B
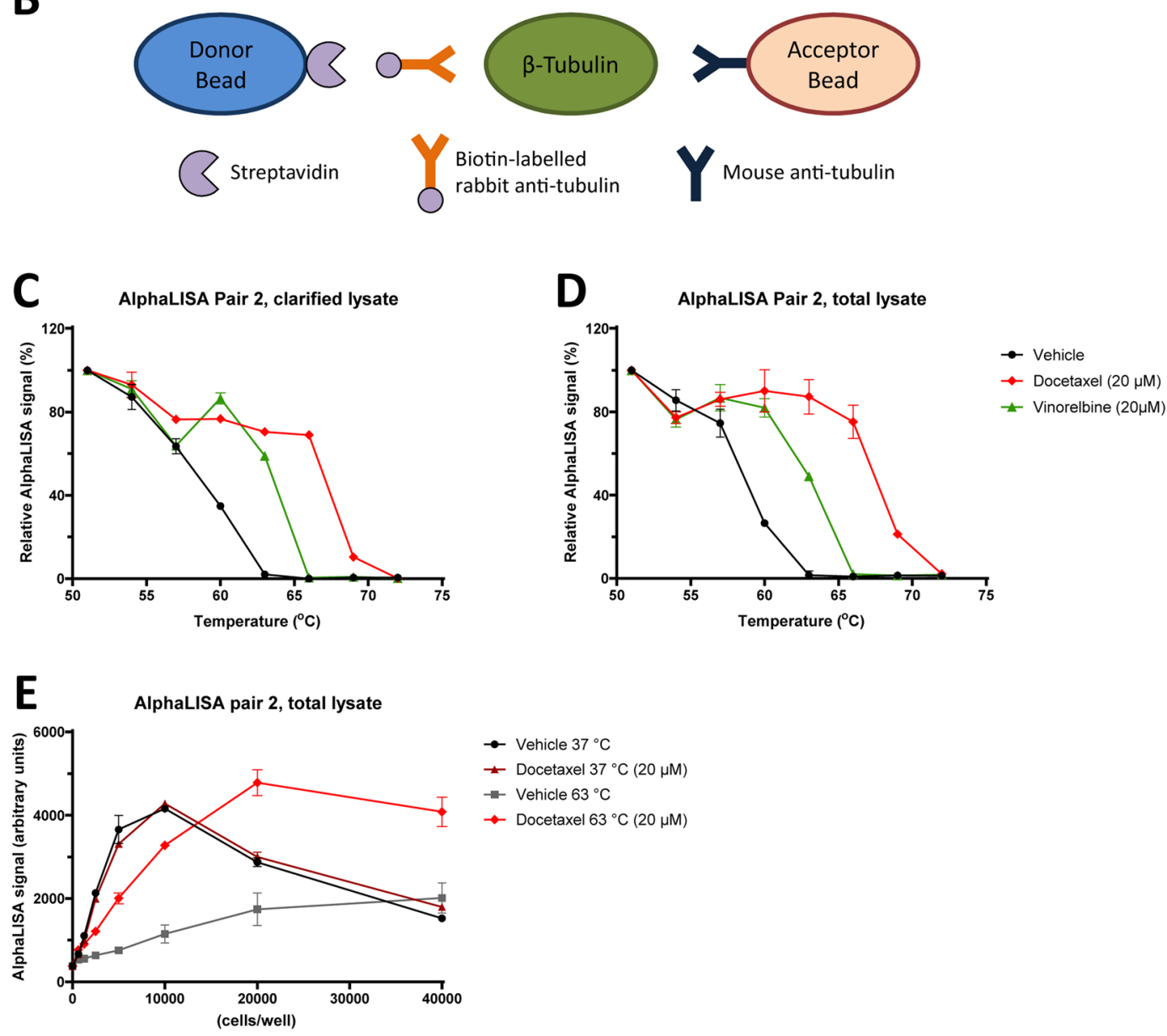

Figure 2. Miniaturization of the $\beta$-tubulin CETSA assay. Schematic drawing of the principle of standard and conjugated AlphaLISA (A and B). CETSA melt curves in K562 cells treated with docetaxel or vinorelbine $(20 \mu \mathrm{M}) . \beta$-tubulin was detected in total and clarified cell lysate with AlphaLISA pair $2(\mathbf{C}$ and $\mathbf{D})$. Cell density titration for AlphaLISA pair 2 in lysate from K562 cells treated with docetaxel $(20 \mu \mathrm{M})$ or vehicle and heated to 37 or $63^{\circ} \mathrm{C}(\mathbf{E})$. The data represent the mean \pm S.E.M from technical replicates and are presented as a percentage of the signal detected at the lowest temperature in each melt curve.

Image-CETSA to monitor TE in specific cell types in heterogeneous samples. For clinical applications, the analysis of TE in individual cells is of high interest since it would facilitate assessment of TE in specific cell types within a heterogeneous cell population existing in one tumour sample. One potential way to accomplish single cell resolution in CETSA is to quantify stabilization by antibody-based cell imaging, using an antibody that specifically recognizes the folded form of the protein. We developed an imaging CETSA format where we investigated several tubulin-directed antibodies and optimized the protocols, resulting in the experimental scheme as shown in Fig. S4A. Starting from cells in suspension, after drug treatment and subsequent CETSA heating in PCR-tubes, cells were washed and collected by centrifugation before being transferred to clear bottom plates where they were fixed and stained (anti-tubulin and Hoechst). After we had developed this CETSA imaging format, two articles describing related methods were published on CETSA drug screening on adherent 

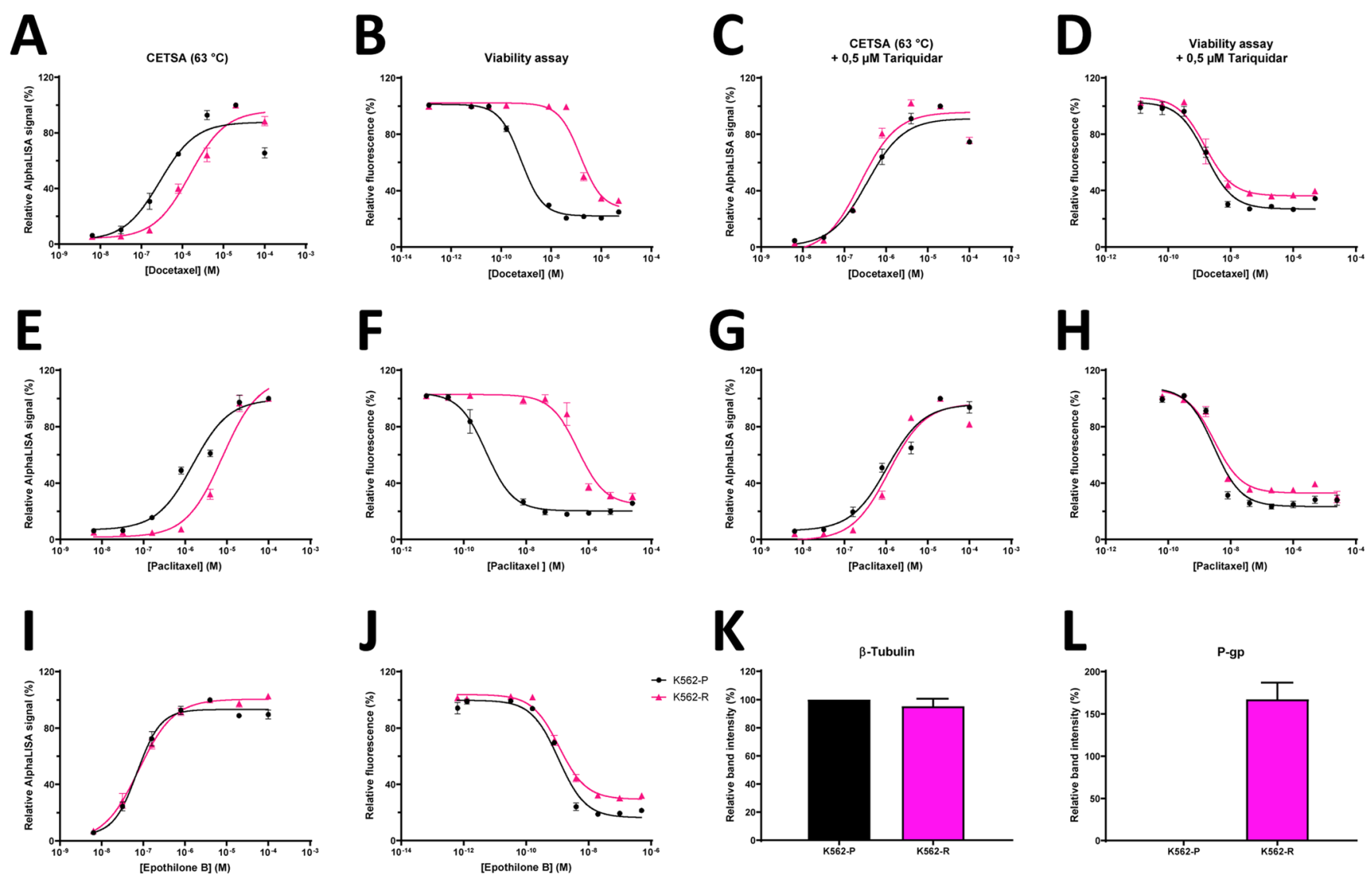

Figure 3. CETSA TE measurements correlate to sensitivity to taxanes and report on the mechanism of resistance. ITDR-CETSA for $\beta$-tubulin at $63^{\circ} \mathrm{C}$ and viability assays in multidrug-resistant (K562-R) and the corresponding parental cells (K562-P) in response to increasing concentrations of docetaxel (A-D) or paclitaxel $(\mathbf{E}-\mathbf{H})$ and in the absence or presence of the Pgp-inhibitor tariquidar $(\mathbf{C}, \mathbf{D}, \mathbf{G}, \mathbf{H})$. ITDRs at $63^{\circ} \mathrm{C}$ and viability assays performed in response to the non-Pgp-substrate epothilone $\mathrm{B}$ (I and $\mathbf{J})$. $\beta$-tubulin was detected with AlphaLISA in the ITDR-CETSA experiments. Pgp- and $\beta$-tubulin-expression in the two cell lines was detected with western blot $(\mathbf{K}, \mathbf{L})$. ITDR-CETSA data are presented as relative to the compound concertation where maximum stabilization is achieved. Cell viability data is relative to the untreated samples. All data represent the mean \pm S.E.M from independent experiments $(n=3-4)$.

cells, demonstrating the feasibility of the image-based detection of target engagement ${ }^{29,30}$. Our method is, however, more versatile and allows for studies of clinical samples of cell suspensions such as fine needle aspirates. Moreover, our protocol allows the use of different temperatures to generate melt curves.

To test our tubulin Image-CETSA implementation for assessing drug efficacy, we first generated melt curves by exposing K562 cells to different temperatures after incubation with and without docetaxel or paclitaxel (Fig. 4A and Table 3). The Image-CETSA melt curves were similar to melt curves produced by western blot CETSA from the same samples and both drugs have apparent stabilization (Figs. S4B and 4A). We then performed ITDR-CETSA experiments, showing that the imaging protocol was able to generate dose response curves (Figs. S4C and 4B). ITDRs with docetaxel were also performed in the resistant K562-R cells comparing them with their parental counterparts K562-P. A shift towards a higher EC $_{50}$ value was observed in the ITDR-CETSA curve corresponding to K562-R cells for both paclitaxel and docetaxel, supporting that Image-CETSA can indeed be used to monitor drug sensitivity (Fig. 4B,C and Table 3). In Fig. 4D, representative images show that Image-CETSA in principle can reveal TE information in individual cells and should be applicable to monitor TE in individual cell types in heterogeneous samples.

Taxane TE in mouse xenografts in ex vivo and in vivo settings. An advantage of CETSA is that the same measurement principle for target engagement can be used in both cell lines and tissues samples. To explore the use of tubulin CETSA in animal models, we used xenografts in mice for ex vivo and in vivo treatment. First, mice with MCF-7 derived xenografts were injected i.v. with drug or vehicle $30 \mathrm{~min}$ before euthanasia, directly followed by tumor sampling and preparation for CETSA analysis. In this first experiment a dose of $50 \mathrm{mg} / \mathrm{kg}$ was used, which is equivalent to $150 \mathrm{mg} / \mathrm{m}^{2}$ for humans ${ }^{31}$, a dose that is somewhat higher than routinely used in the clinic. A significant stabilization of $\beta$-tubulin was observed for $\beta$-tubulin at $60^{\circ} \mathrm{C}$ (Fig. 5A and Table 4 ).

In a second experiment we therefore repeated the treatment with $50 \mathrm{mg} / \mathrm{kg}$ docetaxel in mice bearing MCF-7 xenografts and included two additional docetaxel doses $(33 \mathrm{mg} / \mathrm{kg}$ and $16,5 \mathrm{mg} / \mathrm{kg})$ and two additional 


\begin{tabular}{|c|c|c|c|c|c|}
\hline \multirow[b]{2}{*}{ CETSA } & \multicolumn{2}{|l|}{ K562-P } & \multicolumn{3}{|l|}{ K562-R } \\
\hline & $\begin{array}{l}\text { EC50 and St. } \\
\text { Error }(\mu \mathrm{M})\end{array}$ & $95 \% \mathrm{CI}$ & $\begin{array}{l}\text { EC50 and St. } \\
\text { Error }(\mu M)\end{array}$ & $95 \% \mathrm{CI}$ & Significance* \\
\hline Docetaxel & $0,30 \pm 0,08$ & $0,18-0,5$ & $1,54 \pm 0,25$ & $1,10-2,20$ & $* * *$ \\
\hline Docetaxel $+0,5 \mu \mathrm{M}$ Tariquidar & $0,37 \pm 0,09$ & $0,23-0,58$ & $0,26 \pm 0,07$ & $0,16-0,42$ & ns \\
\hline Paclitaxel & $1,55 \pm 0,3$ & $0,96-2,51$ & $8,34 \pm 1,63$ & $5,90-11,81$ & $* * *$ \\
\hline Paclitaxel $+0,5 \mu \mathrm{M}$ Tariquidar & $1,06 \pm 0,21$ & $0,66-1,72$ & $1,2 \pm 0,27$ & $0,80-1,78$ & ns \\
\hline Epothilone B & $0,08 \pm 0,02$ & $0,06-0,13$ & $0,1 \pm 0,01$ & $0,08-0,14$ & ns \\
\hline Viab. Assay & \multicolumn{2}{|l|}{$(\mathrm{nM})$} & \multicolumn{3}{|l|}{$(\mathrm{nM})$} \\
\hline Docetaxel & $0,64 \pm 0,08$ & $0,50-0,85$ & $150 \pm 30$ & $106-213$ & $* * * *$ \\
\hline Docetaxel $+0,5 \mu \mathrm{M}$ Tariquidar & $1,64 \pm 0,32$ & $1,16-2,32$ & $1,65 \pm 0,35$ & $1,13-2,43$ & ns \\
\hline Paclitaxel & $0,58 \pm 0,14$ & $0,37-1,02$ & $457 \pm 106$ & $306-680$ & $* *$ \\
\hline Paclitaxel $+0,5 \mu \mathrm{M}$ Tariquidar & $3,18 \pm 0,76$ & $2,14-4,72$ & $3,29 \pm 0,69$ & $2,30-4,72$ & ns \\
\hline Epothilone B & $1,1 \pm 0,17$ & $0,83-1,44$ & $1,2 \pm 0,16$ & $0,92-1,53$ & ns \\
\hline
\end{tabular}

Table 2. Overview of $\mathrm{EC}_{50}$ values with associated standard errors and $95 \%$ confidence intervals (CI), and statistical significance calculated for the ITDR-CETSA curves in Fig. 3A-J. *The significance was determined for K562-R vs. K562-P using a two-tailed T-test for $\mathrm{EC}_{50}$ values from independent experiments. P-values ns $\mathrm{P}>0.05, * \mathrm{P}<0.05, * * \mathrm{P}<0.01, * * * \mathrm{P}<0,001, * * * * \mathrm{P}<0,0001$ compared to K562-P.

temperatures for the melt curves. This is equivalent to human doses of $100 \mathrm{mg} / \mathrm{m}^{2}$ and $50 \mathrm{mg} / \mathrm{m}^{2}$, which are commonly used in monotherapy and combination treatment respectively. A significant stabilization of tubulin was observed also with lower doses of docetaxel, demonstrating that CETSA-based TE can potentially be detected at clinically relevant doses (Fig. 5B and Table 4).

For comparing local tumor cell effects of different drugs, an ex vivo setting experiment is preferred, since this format would allow to evaluate target binding of multiple drugs in e.g. the same patient biopsy. To test this setting and an additional xenograft model, ex vivo experiments were performed in MDA-MB-231 (triple negative breast cancer cell line) derived xenograft tumors. Ex vivo exposure to $50 \mu \mathrm{M}$ docetaxel also showed a very prominent shift, albeit with larger standard deviations than in the in vivo treated MCF-7 samples (Fig. 5C and Table 4). Possibly, the larger standard deviations could be due to poorer drug penetration in the solid tumor MDA-MB-231 pieces in the ex vivo setting and indicate that analysis of tissue samples with ex vivo drug treatment should be done using cell suspensions obtained by digesting solid biopsies, rather than by treating pieces. Alternatively, to avoid digestion of tumor tissue, a process that could potentially affect cell characteristics, the tissue could be freshly sliced using a vibratome before incubating with drug.

Characterization of resistance in prostate cancer PDX models. As previously mentioned, an attractive setting to predict clinical outcome of drug treatment is to examine drug response ex vivo in biopsy samples. To investigate if drug TE measured with CETSA in ex vivo experiments could predict in vivo biology of the same drug, we performed experiments using patient-derived xenograft (PDX) based models of tumour drug resistance in castration-resistant prostate cancer. To study taxane sensitivity in resistant models we used two PDX models, PC346C and PC339, and their docetaxel-resistant counterparts PC346C-DOC and PC339-DOC, previously described by de Morrée et al. ${ }^{32}$. Relevant details regarding the abovementioned PDX models are summarized in Table S1A. We first compared the effect of docetaxel, cabazitaxel, paclitaxel, and vinorelbine in cell lines corresponding to the PDX models PC346C and PC339. We found a pronounced difference in potency between the drugs in both cell lines with cabazitaxel as the most potent drug, followed by docetaxel, paclitaxel, and vinorelbine as the least potent drug (Figs. 6A, S5A and Table S1B). The observed ranking correlates with previous studies showing cabazitaxel is more efficient than docetaxel in inhibiting cell proliferation and suppression of microtubule dynamics ${ }^{33}$.

Studies of the PC346C and PC346C-DOC cell lines support that the taxane-resistant cells have attenuated TE in response to docetaxel, cabazitaxel, and paclitaxel (Fig. 6B-D and Table S1D) when both EC50s and maximum response levels are affected, reiterating the published in vivo results showing that taxane uptake is strongly impaired in the PC346C-DOC model.

PC346C-DOC express less of the influx transporter SLCO1B3, as compared to the parental counterpart PC346C, which is shown to result in almost fully depleted intratumoural taxane levels ${ }^{34}$. The effect of cabazitaxel on TE was more pronounced than that of docetaxel in both PC364C and PC346C-DOC tumor slices, which is in line with its reported higher efficacy ${ }^{35}$.

Similar studies of PC339C and PC339C-DOC do not show any significant difference between the cell lines, consistent with that processes effecting residual target engagement are not altered in this model.

Subsequently, ex vivo treatments of PC346C and PC339 PDX tumors (freshly sliced using a vibratome) with increasing concentrations of docetaxel and cabazitaxel were performed. Standard deviations were high for these experiments for technical reasons, as discussed below, but the results support that maximum $\beta$-tubulin TE in PC346C-DOC compared to the parental counterpart PC346C after both docetaxel and cabazitaxel treatment (Fig. 6E) is changed in a similar manner as in the cell lines. In contrast, PC339 and the docetaxel resistant variant PC339-DOC did not show effects on TE (Fig. S5E). Previous data show PC339-DOC to remain sensitive to cabazitaxel and that the docetaxel resistance in this model is possible to overcome by increased dosing of docetaxel ${ }^{32}$. 
A

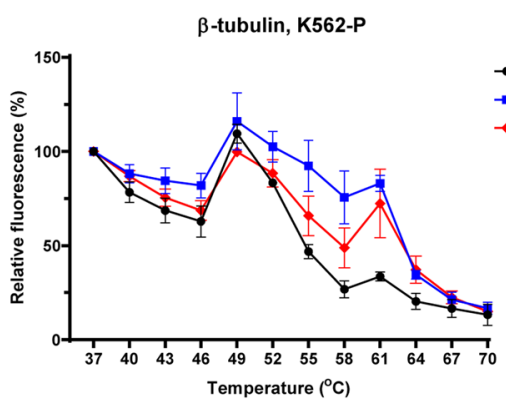

C

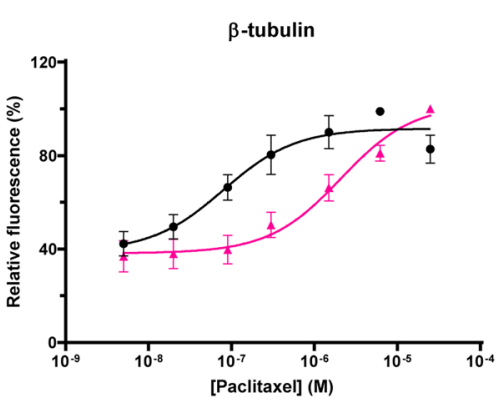

B

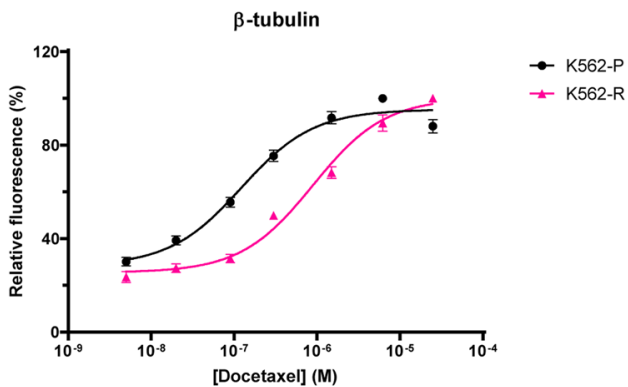

D
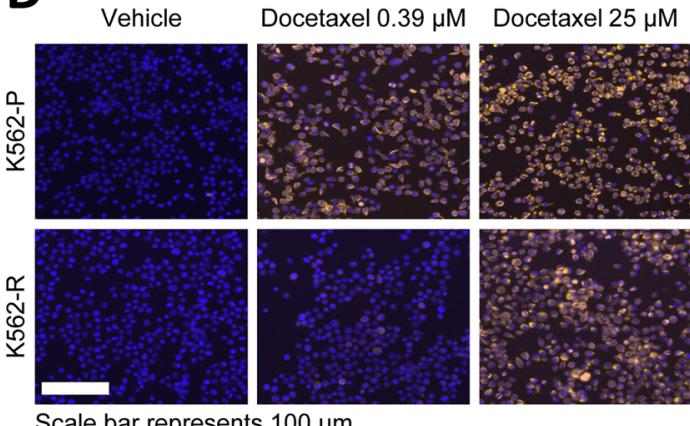

Figure 4. Imaging-CETSA has the potential of quantifying TE in individual cells and report on cellular resistance. Imaging-CETSA melt curves for $\beta$-tubulin in response to docetaxel and paclitaxel $(5 \mu \mathrm{M})(\mathbf{A})$. ITDRCETSA in multidrug-resistant (K562-R) and parental (K562-P) cells after exposure to different concentrations of docetaxel (B) or paclitaxel (C) and heating at $56^{\circ} \mathrm{C}$. Representative images of K562-P and K562-R cells treated with docetaxel or vehicle (D). Hoechst staining of the nuclei is shown in blue while $\beta$-tubulin staining is shown in yellow. All data represent the mean \pm S.E.M from independent experiments $(n=3-4)$ and are presented as a percentage of the signal detected at the lowest temperature in each melt curve (A) or maximum stabilization detected in each series (B and $\mathbf{C}$ ).

\begin{tabular}{|c|c|c|c|}
\hline \multirow[b]{2}{*}{ Docetaxel } & \multicolumn{3}{|c|}{ Imaging-CETSA } \\
\hline & $\begin{array}{l}\text { EC50 and St. } \\
\text { Error }(\mu M)\end{array}$ & $95 \% \mathrm{CI}$ & Significance* \\
\hline K562-P & $0,12 \pm 0,02$ & $0,09-0,16$ & - \\
\hline K562-R & $0,83 \pm 0,12$ & $0,60-1,17$ & $* *$ \\
\hline Paclitaxel & $\begin{array}{l}\text { EC50 and St. } \\
\text { Error }(\mu M)\end{array}$ & $95 \% \mathrm{CI}$ & Significance* \\
\hline K562-P & $0,08 \pm 0,04$ & $0,03-0,20$ & - \\
\hline K562-R & $2,00 \pm 0,83$ & $0,73-5,41$ & $* *$ \\
\hline
\end{tabular}

Table 3. Overview of $\mathrm{EC}_{50}$ values with associated standard errors and $95 \%$ confidence intervals (CI), and statistical significance calculated for the ITDR-CETSA curves in Fig. 4B,C. *The significance was determined for K562-R vs. K562-P using a two-tailed T-test for $\mathrm{EC}_{50}$ values from independent experiments. P-values ns $\mathrm{P}>0.05, * \mathrm{P}<0.05, * * \mathrm{P}<0.01, * * * \mathrm{P}<0,001, * * * * \mathrm{P}<0,0001$ compared to K562-P.

These results support that the mechanism of resistance in PC339-DOC is to be downstream of, or alternatively bypassing, target engagement. Taken together, our data support that CETSA TE studies can differentiate between sensitive and resistant tumors in the cases where taxane resistance occurs up to the level of TE, for example in the case of altered expression of membrane transporters.

Drug TE measurements in fine needle aspirates from breast cancer patients. As shown above in several model systems, tubulin CETSA for taxanes generally correlates with drug sensitivity. To establish and evaluate the method for clinical samples we used fine needle aspirates (FNAs), since previous data indicates that measurements in cell suspensions tend to have lower variation than tissue pieces and that tumor cells are highly enriched in FNA samples ${ }^{36}$. We first tested the amenability of using FNAs by taking such samples from mouse xenografts. CETSA stabilization upon ex vivo treatment with $25 \mu \mathrm{M}$ docetaxel of FNAs from MCF-7 xenografts was detected by AlphaLISA and shown to be linear for several cell concentrations (Fig. 7A). 

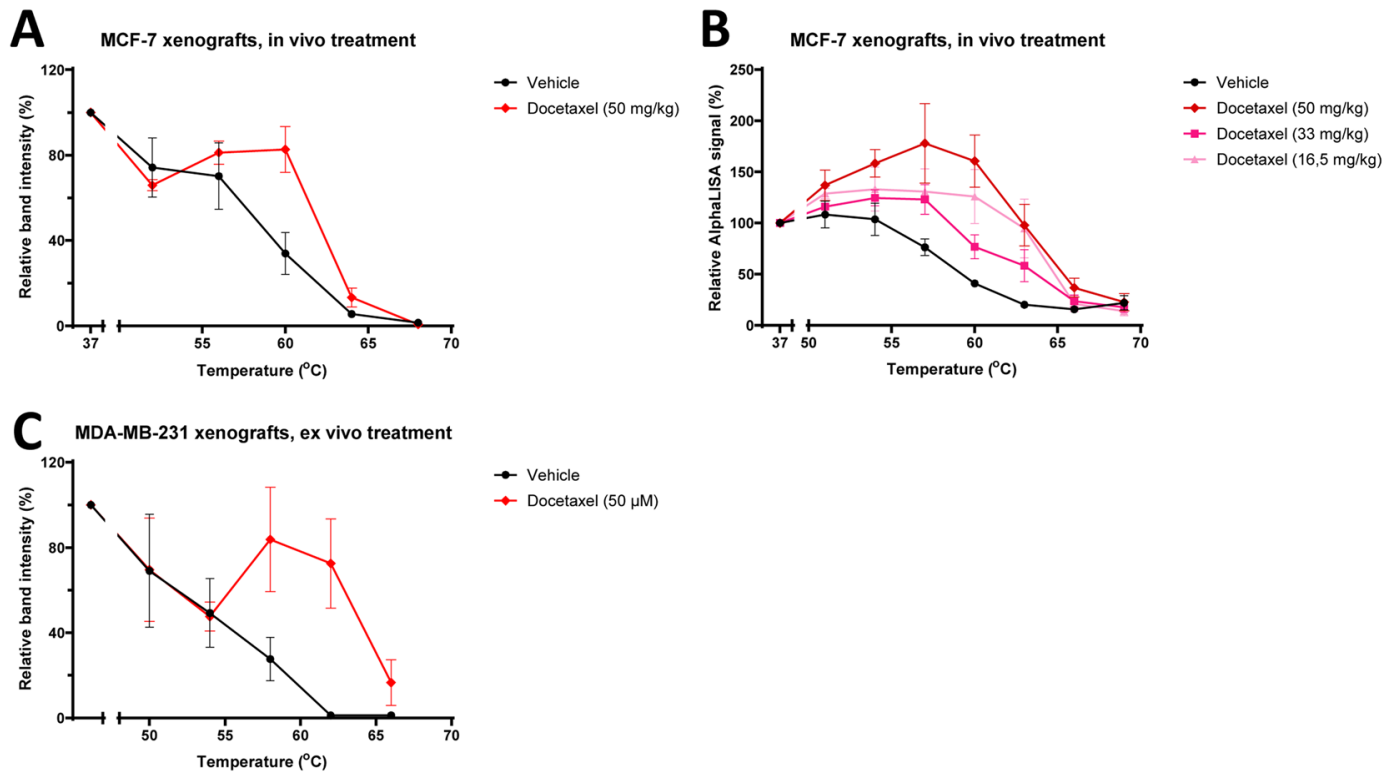

Figure 5. Docetaxel produces CETSA shifts for $\beta$-tubulin in both in vivo and ex vivo mice models. SCID-mice bearing MCF-7 xenograft tumours were treated in vivo for $30 \mathrm{~min}$ with docetaxel at a dose of $50 \mathrm{mg} / \mathrm{kg}$ before being sacrificed and the tumours taken for $\beta$-tubulin analysis with western blot-CETSA (A). SCID-mice bearing MCF-7 xenograft tumours were treated in vivo with different doses of docetaxel and samples were analysed with AlphaLISA (B). Pieces of MDA-MB-231 xenografts were treated ex vivo with Docetaxel $(50 \mu \mathrm{M})$ before $\beta$-tubulin analysis with western blot-CETSA $(\mathbf{C})$. All data represent the mean \pm S.E.M from different tumours in each condition ( $n=2-3$ in $A$, and $n=4$ in $\mathbf{B}$ and $C$ ) and are presented as a percentage of the signal detected at the lowest temperature in each melt curve.

\begin{tabular}{|l|l|}
\hline MCF-7 Xenografts & $\beta$-tubulin \\
\hline in vivo $\left(60^{\circ} \mathrm{C}\right)$ & Significance* \\
\hline Vehicle & - \\
\hline Docetaxel $(50 \mathrm{mg} / \mathrm{kg})$ & $*$ \\
\hline in vivo $\left(60^{\circ} \mathrm{C}\right)$ & Significance* \\
\hline Vehicle & \\
\hline Docetaxel $(16,5 \mathrm{mg} / \mathrm{kg})$ & $*$ \\
\hline Docetaxel $(33 \mathrm{mg} / \mathrm{kg})$ & ns \\
\hline Docetaxel $(50 \mathrm{mg} / \mathrm{kg})$ & $* *$ \\
\hline ex vivo $\left(62^{\circ} \mathrm{C}\right)$ & Significance* \\
\hline Vehicle & - \\
\hline Docetaxel $(50 \mu \mathrm{M})$ & $*$ \\
\hline
\end{tabular}

Table 4. Overview of the statistical significance of the measured CETSA shift for the data presented in Fig. 5A-C. *The significance of the CETSA shifts was calculated using one-way ANOVA. Adjusted P-values ns P $>0.05$, $* \mathrm{P}<0.05, * * \mathrm{P}<0.01, * * * \mathrm{P}<0,001, * * * * \mathrm{P}<0,0001$ compared to vehicle.

Next, the applicability of CETSA for patient material was assessed by performing experiments on FNAs collected from surgically resected primary tumors from breast cancer patients that have not received any previous treatment. All FNAs were exposed to docetaxel for $15 \mathrm{~min}$, an incubation time that was shown to be sufficient to give full stabilization (Fig. S2F) and yet short enough to have minimal impact on cell viability as well as minimizing potential effect of transcription or other alterations that might occur in cells after being removed from their original environment. A major challenge with this approach was however the low cell number and viability typically obtained from the FNAs, although this varied very significantly between samples (Fig. S6). FNAs containing enough material (at least 10000 live cells per experiment for detection with conjugated beads, and 100000 per experiment for detection with standard AlphaLISA) were subjected to docetaxel treatment and subsequent CETSA analysis. Clear stabilization of $\beta$-tubulin could be detected in most samples analyzed and variability was acceptable between technical replicates. In cases where a sufficient number of viable cells were obtained, FNA samples were incubated with several doses of docetaxel. Surprisingly, these experiments revealed large differences at TE level between patients, up to 100 times (Fig. 7B and Table S2).

FNAs were also collected by cytological aspiration directly from the untreated primary tumours of breast cancer patients. In this case, the viability of the cells was somewhat higher with a mean of $43 \%$ cell viability compared 

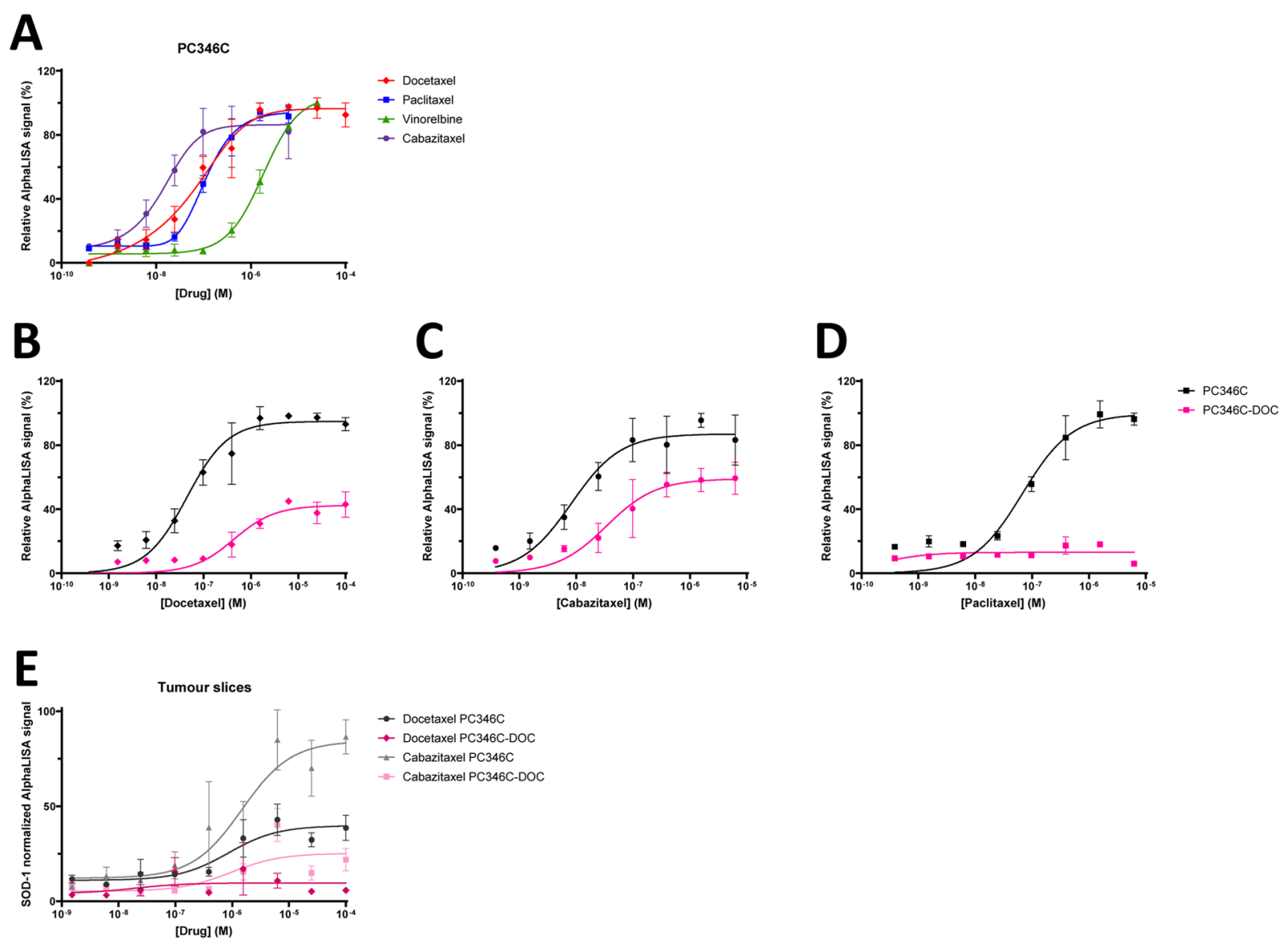

Figure 6. CETSA TE levels correlate with sensitivity to taxanes and reported resistance mechanisms in mouse PDX models of prostate cancer. PDX-derived cell line PC346C was treated with different concentrations of tubulin-binding drugs and $\beta$-tubulin TE was analysed with AlphaLISA (A) PC346C cells and the corresponding resistant cell line PC346C-DOC were treated with different concentrations of taxanes (B-D). Tumours from the PDX model PC346C and the resistant counterpart PC346C-DOC were treated ex vivo with increasing doses of docetaxel and cabazitaxel (E). Data from tumours slices were normalized to SOD-1 levels. All data represent the mean \pm S.E.M from either different tumors in each condition (E) or from independent experiments ( $n=3$ in $\mathbf{A}-\mathbf{D})$.

to $16 \%$ in the FNAs from surgically removed tumors (Fig. S6). This improvement in cell viability may have been the result of the shorter time between FNA collection and performing the CETSA analysis. When FNAs were collected from surgically removed tumours this was typically done at least 1 hour after time of ischemia, which could have contributed to the differences in viability. The assay quality was, however, similar when analyzing samples taken directly from patients and from surgically removed tumours. Again, samples showed a dose dependent response to docetaxel but the efficacy varied between patients (Fig. 7C). K562 cells were treated and analyzed in parallel in each experiment as a technical control of the experiment and for confirming the reproducibility of the AlphaLISA assay. The K562 control experiments resulted in ITDR-CETSA curves for docetaxel with very similar EC50 values for all tested samples (data not shown).

\section{Discussion}

In the present work we establish different formats of CETSA to assess target engagement of taxanes, and other tubulin directed drugs, to microtubules in cells and tissues, including breast cancer patient FNAs. The different CETSA formats give consistent measurements of melting behavior and ITDRs, although some quantitative differences are seen in melting curve shapes. We show that melt curves are stabilized by several tubulin modulators binding at both the vinca and the taxane site on $\beta$-tubulin. The very stabilization by vinorelbine is surprising and indicates that this family of drugs, in addition to inhibiting tubulin polymerization, also have a dramatic effect in intrinsic microtubule stability, which might add significantly to their cytotoxic mechanism. The optimized AlphaLISA assays provide a miniaturized and sensitive format allowing for measurements using relatively small number of cells (1000 cells per measurement point). Miniaturized and sensitive CETSA measurements are particularly valuable when many measurements points (replicates, doses, time points, etc.) are assessed in model studies, and sensitivity is essential for measurements on biopsy material where low cell number is often the limiting factor. The imaging CETSA implementation provides an interesting alternative, when different cell types can be selected from images, and TE can in principle be assessed in each cell type (although this was not directly tested in this study). 

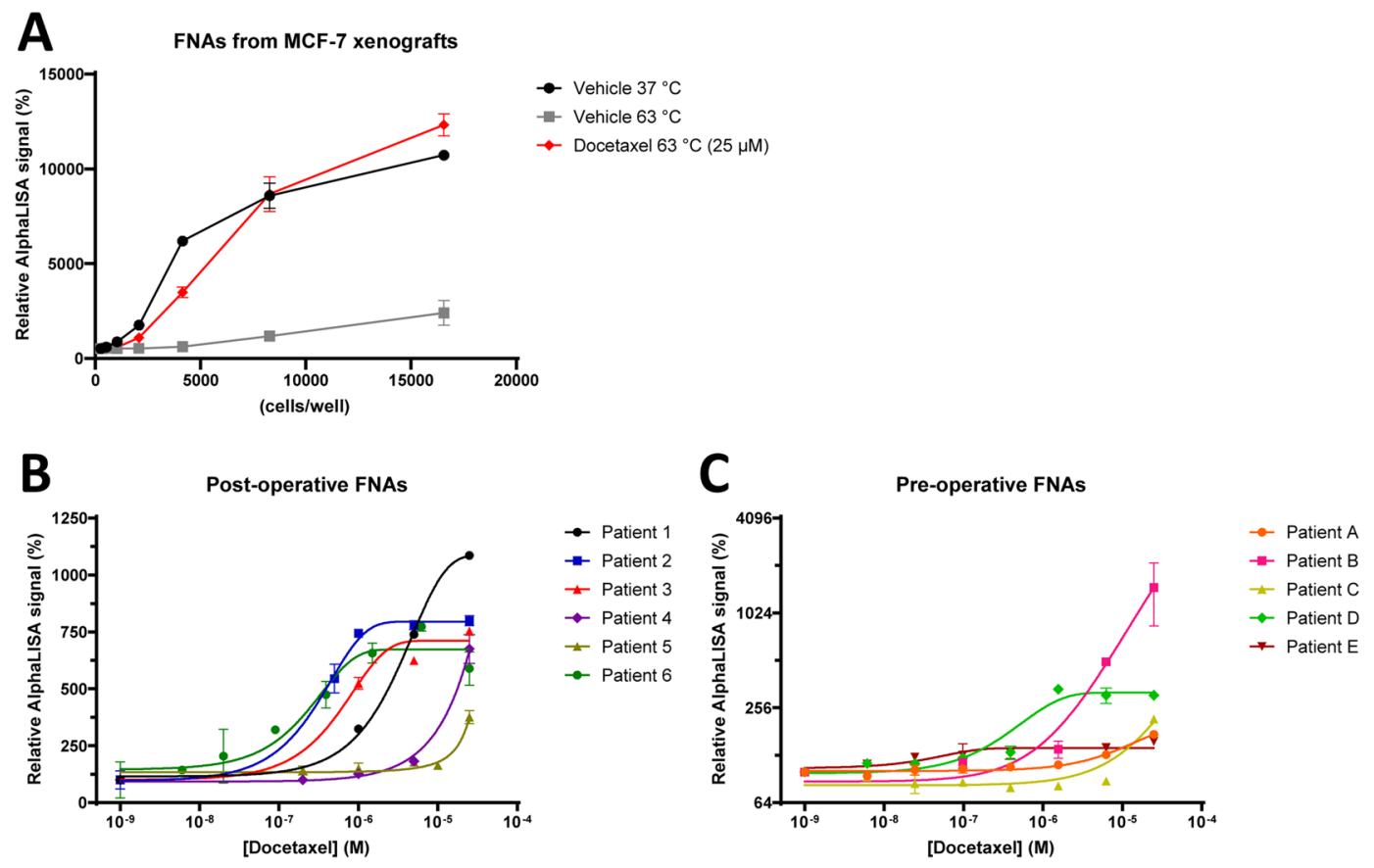

Figure 7. CETSA for assessing TE for taxanes in ex vivo-treated breast cancer patient FNAs. Fine needle aspirates (FNAs) were taken from freshly removed MCF-7 xenografts and treated with docetaxel $(25 \mu \mathrm{M})$ for $15 \mathrm{~min}$ before CETSA-heating at 37 or $63^{\circ} \mathrm{C}$. $\beta$-tubulin for different lysate concentrations was analysed with AlphaLISA (A). Fine needle biopsies from surgically removed tumours (B) or directly from patients (C) were treated with different concentrations of docetaxel for $15 \mathrm{~min}$ before CETSA-heating at $63^{\circ} \mathrm{C}$ and $\beta$-tubulin analysis with AlphaLISA. The data represent the mean \pm S.E.M from technical replicates and are presented as a percentage of the signal detected in the vehicle-treated samples.

A specific challenge of the tubulin specific assay is the unusually high melting temperature of tubulin in intact cells, being in the range where some cells are prone to heat induced lysis. Fortunately, tubulins are depolymerized when cells lyse and do not bind taxanes. Furthermore, tubulin in lysed cells melt some $10^{\circ} \mathrm{C}$ earlier than in intact cells, so no protein from lysed cells is detected at the temperature used for most of the ITDRs $\left(63^{\circ} \mathrm{C}\right)$.

Most studies of cancer drug sensitivity is done using cell viability assays with durations of $48-72 \mathrm{~h}$, but such assays are typically hard to establish in reproducible formats for material from solid tumors. Also, different cell programs and distribution of different cancer cell clones in the sample can change very significantly during extended culturing, as compared to the situation in the tumor. An advantage with the ex vivo CETSA assay is that it allows for rapid measurement of TE, where fresh tumor samples are incubated with different drugs for short time periods (15-120 minutes), during which cell programs and cell clone distributions are less affected. On the other hand, CETSA only assesses cellular responses up to the target engagement level. For tubulin-directed drugs, however, several mechanisms proposed so far for drug resistance include the level of effective intracellular drug concentrations (through changes in influx, efflux, or catabolism) as well as modulation of tubulin structure (e.g. tubulin mutations or overexpression of beta-III-tubulin) ${ }^{12-14}$, which should be directly accessible with CETSA measurements.

In support that CETSA-derived measurement of tubulin binding correlates well with cell toxicity we show that CETSA ITDRs reflect known ranking of toxicities of different tubulin binding drugs in cell lines. When toxicity of tubulin inhibition is primarily expected in M-phase, this suggests that drug binding and buildup of effective drug concentration in other cell cycle phases, that dominate our samples, correlates well with the situation in M-phase. Importantly, CETSA directly reflects the difference between parental and resistant cells as shown for the multidrug resistant $\mathrm{K} 562$ cells as well as for PC346C-DOC taxane-resistant prostate cancer.

In the PC339-DOC PDX model TE was retained, consistent with the known increased drug accumulation of cabazitaxel in the resistant cells. This supports a bypass, or downstream mechanism of acquired resistance for this PDX model which remains to be characterized. This illustrates that even in cases were CETSA reveals TE not to be affected, such information will be valuable for dissecting resistance mechanisms for cancer drugs, since CETSA captures both effective intracellular drug concentrations and target modifications. In cases where TE is not changed in resistant cells, focus can instead be put on discovering downstream mechanisms for resistance. The mass-spectrometry implementation of CETSA (MS-CETSA) now provides a novel mean to dissect downstream/bypass mechanisms for drug resistance involving modulations of protein interaction states ${ }^{26}$.

Clinical evaluation of tubulin CETSA is outside the scope of this work and remains to be done for the validation of the usefulness of the assay to predict clinical outcome and to stratify drug selection. The present work does, to the best of our knowledge, for the first time present CETSA data for patient samples revealing variations in dose response. When tubulin CETSA data show correlation with drug toxicity in models it has the potential to be useful as an early prognostic biomarker to detect the emergence of resistance for different drugs up to the TE 
level in patient biopsies, where the cross resistance to alternative tubulin directed therapies can also be accessed. It is likely that drug targets for many other clinical drugs are similarly applicable for CETSA and that the resistance of other classes of drugs in combination therapies can be accessed in parallel with tubulin-specific acquired resistance. In the clinical situation, it has previously been difficult to pin down acquired resistance for individual drugs in combination regimes, except in cases where information on specific mutational patterns leading to target protein modifications has been available.

In conclusion, we have established and validated several protocols for measuring tubulin-specific CETSA of taxanes in cell lines and mouse models, demonstrating that drug effects up to the level of TE can be rapidly assessed in cells and tissues. Correlation of CETSA-based detection of TE with cell toxicity in several of the tested models support that CETSA measurements can provide a novel and valuable alternative in the clinical setting to rapidly generate actionable information for therapeutic decisions. Experiments on FNAs from breast cancer patients demonstrate that CETSA TE can indeed be measured in some patient samples with sufficient accuracy in the ex vivo setting. The relatively broad range of responses on the TE level seen in these non-treated patients, suggest varying drug sensitivity and potentially that initial resistance can be detected in patients. To rapidly assess the relative efficacy of several tubulin directed drugs could therefore help in stratification of next line therapies. However, in spite of the high sensitivity of the miniaturized assays, for many patients the number of viable cells were not sufficient from a single FNA (Fig. S6) suggesting that e.g. core biopsies, and more sensitive detection methods are needed for CETSA measurements in a majority of patients.

\section{Materials and Methods}

Drugs. Docetaxel (S1148), paclitaxel (S1150), vinorelbine (S4269), epothilone B (S1364), daunorubicin (S3035), and cytarabine (S1648) were obtained from Selleckchem. All drugs were solubilized in DMSO at a concentration of $50 \mathrm{mM}$ and stored frozen until use. Drugs were generally diluted 4 times in DMSO before further dilution in HBSS or media.

Cell culture. All cell lines were cultured in $5 \% \mathrm{CO}_{2}$ and $37^{\circ} \mathrm{C} . \mathrm{K} 562$ (ATCC-CCL-243) was grown in RPMI (Sigma R8768), SK-BR-3 (ATTCC-HTB-30) in McCoy's 5a media (Sigma), and MCF-7 (ATCC-HTB-22) and MDA-MB-231 (ATCC-HTB-26) were grown in DMEM high glucose (Sigma D6429). All media were supplemented with 10\% heat inactivated fetal bovine serum (FBS) (Gibco 10500-064) and antibiotic-antimycotic (Life technologies 15240-062). Multidrug-resistant and parental K562 cells were kindly provided by Sören Lehmann and Christer Paul at the Karolinska Institute. Cells were passaged every second to third day. For adherent cells passaging included washing the cells in HBSS (Life technologies $14175-053-\mathrm{CaCl}_{2},-\mathrm{MgCl}_{2}$ ) and detaching in TrypLE (Life Technologies 12563-029). The PC346C and PC339C cell lines were cultured in prostate growth media (PGM) as described in ${ }^{37}$ and the docetaxel-resistant counterparts were cultured in the presence of $0.1 \mathrm{nM}$ docetaxel. The multidrug-resistant K562-cells were incubated for 72-96 hours with $150 \mathrm{nM}$ vincristine (or DMSO for the parental cells) once a week.

CETSA melt curves and ITDR-CETSA with intact cells. Cells were harvested, washed, and diluted in HBSS (Life technologies $14025100+\mathrm{CaCl}_{2},+\mathrm{MgCl}_{2}$ ) to 4 million cells $/ \mathrm{ml}$ for western blot and 3,33 million cells $/ \mathrm{ml}$ for AlphaLISA. The cell suspension was mixed with drug or vehicle before aliquoting into PCR-tubes and kept at $37^{\circ} \mathrm{C}$ for $1 \mathrm{~h}$ unless otherwise indicated. During longer incubations, tubes were rotated every $20 \mathrm{~min}$. CETSA heating for $3 \mathrm{~min}$, unless otherwise stated, was performed in a Veriti Thermal cycler (Applied Biosystems), either at a range of temperatures for melt curves or at $63^{\circ} \mathrm{C}$ for ITDRs unless otherwise specified. Cells were subsequently lysed by three rounds of freeze-thawing by alternating exposure of the samples to liquid nitrogen and $20^{\circ} \mathrm{C}$ in a PCR-machine. For western blot experiments the aggregated proteins were removed by centrifugation at $20000 \mathrm{~g}$ for $20 \mathrm{~min}$ at $4^{\circ} \mathrm{C}$ and the supernatant was collected. Samples were either analyzed directly or stored at $-80^{\circ}$.

CETSA melt curves with cell lysate. Cells were harvested, washed, and diluted in HBSS (Life technologies $14025100+\mathrm{CaCl}_{2},+\mathrm{MgCl}_{2}$ ) to 40 million cells $/ \mathrm{ml}$ and freeze-thawed three times, as described above, with vortexing in between for proper cell lysis. The high cell density was used in order to obtain a protein concentration more similar to that inside intact cells. The lysates were centrifuged at $20000 \mathrm{~g}$ for $20 \mathrm{~min}$ at $4{ }^{\circ} \mathrm{C}$, the supernatant collected, and the clarified lysate was stored at $-80^{\circ}$ until use. For the experiments, the lysate was mixed with drug or vehicle and incubated in PCR-tubes for $10 \mathrm{~min}$ at RT. CETSA heating and centrifugation were performed as described above. Before western blot analysis the samples were diluted to $5 \mathrm{x}$ in HBSS before mixing with the western blot loading buffer in order to get the same protein concentration in the samples as used for the intact-cell experiments.

Western blot. Samples were mixed with NuPage loading buffer consisting of NuPage LDS sample buffer (Life technologies NP008) and reducing agent (Life technologies NP009) and vortexed. Proteins were separated on a Bis-Tris $4-12 \%$ polyacrylamide midi gel (Invitrogen WG1403BX10) for $45-50 \mathrm{~min}$ at $200 \mathrm{mV}$. Directly after the run the gels were washed in deionized water and transferred to nitrocellulose membranes using the iBlot 2 system (Invitrogen) and iBlot 2 NC Regular stacks (Invitrogen IB23001). Membranes were washed for $10 \mathrm{~min}$ in TBS with 0,05\% Tween 20 (Medicago 09-7510-100) (TBS-T) and blocked in 5\% (w/v) non-fat milk (Semper AB) in TBS-T for $1 \mathrm{~h}$ before incubation with primary antibody over night at $4{ }^{\circ} \mathrm{C}$ with gentle shaking. After washing in TBS-T for $5 \times 5 \mathrm{~min}$, membranes were exposed to secondary antibodies for $1 \mathrm{~h}$, washed again $5 \times 5 \mathrm{~min}$ in TBS-T and developed using Clarity Western ECL Substrate (BioRad \#170-5061). The chemiluminescent signal was detected using the ChemiDoc ${ }^{\mathrm{TM}}$ XRS + imaging system from BioRad and band intensities were quantified using ImageLab ${ }^{\mathrm{TM}}$ software (BioRad). 
Primary antibodies used were T5201 from Sigma for $\beta$-tubulin, sc-32293 from Santa Cruz for $\alpha$-tubulin, and HPA001401 from Sigma or sc-11407 from Santa Cruz for SOD-1. When comparing western blot with Image-CETSA the $\beta$-tubulin antibody \#2128 from Cell Signalling was used since that is the same clone (9F3) as the Alexa Fluor 488 conjugated antibody \#3623 that was used for Image-CETSA.

Secondary antibodies used were either sc-2055 from Santa Cruz or W402B from Promega for mouse and sc-2374 from Santa Cruz or W401B from Promega for rabbit. All antibodies were diluted in 5\% (w/v) non-fat milk in TBS-T.

Trypan blue exclusion. In order to monitor membrane integrity after heating, cells were harvested according to the same protocol as for the CETSA experiments (see above) and heated in the Veriti Thermal cycler according to the indicated temperatures and time points. After heating, a $10 \mu \mathrm{l}$ aliquot was immediately taken and mixed with $10 \mu \mathrm{l} 0.4 \%$ Trypan Blue stain (Gibco/Life Technologies 15250-061). $10 \mu \mathrm{l}$ of this mix was directly loaded on a counting slide (BioRad \#145-011) and read in an automated cell counter (BioRad TC20).

Standard AlphaLISA assay. For detection with AlphaLISA, $3 \mu$ of each sample was loaded in duplicates in an AlphaPlate (Perkin Elmer 6008350) followed by addition of $2 \mu$ l antibody mix. Two different combinations of antibodies were used: either the mouse-anti-tubulin antibody sc-398937 (raised against amino-acids 209305 within an internal region of human beta4-tubulin, Santa Cruz) in combination with the rabbit-anti-tubulin antibody ab6046 (raised against a synthetic peptice corresponding to human beta tubulin amino-acids 1-100, Abcam) referred to pair 2, or pair 8 where the mouse antibody was changed to T5201 (recognizes all five isoforms of beta-tubulin and binds to the carboxy-terminal part, Sigma). Sc-389837 was diluted to $45 \mathrm{nM}$ in order to get the final concentration of $1 \mathrm{nM}$, T5201 to $135 \mathrm{nM}$ to get final concentration of $3 \mathrm{nM}$, and ab6046 was diluted to $450 \mathrm{nM}$ to get $10 \mathrm{nM}$ in the final mix. All dilution was made in AlphaLISA Immunoassay Buffer (Perkin Elmer AL000F). Anti-rabbit acceptor beads (Perkin Elmer AL104M) and anti-mouse donor beads (Perkin Elmer AS104D) were diluted in the same AlphaLISA buffer to $22.5 \mu \mathrm{g} / \mathrm{ml}$ and $90 \mu \mathrm{g} / \mathrm{ml}$ respectively to get $10 \mu \mathrm{g} / \mathrm{ml}$ and $40 \mu \mathrm{g} / \mathrm{ml}$ as final concentration when adding $4 \mu \mathrm{l}$ of the mix to each well. All handling of the beads was done in a room with green light. Plates were sealed with sticky film, covered in foil, and spun at $177 \mathrm{rcf}$ for 1-3 min. After overnight incubation in the dark, plates were spun briefly at $300 \mathrm{rcf}$ and read in an Enspire 2300 (Perkin Elmer).

Conjugation of AlphaLISA beads. Two antibody pairs were conjugated: ab6046 (abcam) and T5201 (Sigma) for tubulin (pair 8) and the polyclonal AF3418 for SOD-1. The SOD-1 antibody AF3418 (R\&D Systems) was divided in two aliquots of which one was biotinylated and one was conjugated to the Acceptor beads (Perkin Elmer 6772001). For $\beta$-tubulin ab6046 (abcam) was biotinylated and T5201 (Sigma) was used for conjugation to Acceptor beads. Biotinylation of respective antibody were performed using an antibody biotinylation kit (Pierce/ Thermo scientific 90407).

Before performing biotinylation, the buffer of ab6046 needed to be exchanged to PBS which was done using Zeba spin column (Thermo Scientific 89882) according to the manufacture's instructions. Then $100 \mu \mathrm{PBS}$ was added to one tube of NHS-PEG4-Biotin and mixed by pipetting. A 40-fold excess of biotin was added to the antibodies, mixed, and incubated for $30 \mathrm{~min}$ at RT. After incubation, the buffer was exchanged using the Zeba spin column (Thermo Scientific 89882) according to manufacturor's protocol. Biotinylated antibodies were aliquoted and stored at $-20^{\circ} \mathrm{C}$ before use.

The antibodies T5201 and AF3418 were conjugated to Acceptor beads (Perkin Elmer 6772001). $0.033 \mathrm{mg}$ beads were used for $0.333 \mathrm{mg}$ antibody. First, the Acceptor beads were washed by adding $17 \mu \mathrm{l}$ PBS (Life technologies 10010), centrifuged at $16000 \mathrm{rpm}$ for $15 \mathrm{~min}$, supernatant discarded, and the pellet dissolved in at mix with $0.033 \mathrm{mg}$ antibody and $130 \mathrm{mM}$ sodium phosphate puffer $(\mathrm{pH} 8.0)$ in a final volume of $67 \mu \mathrm{l}$. In later conjugations, this volume was reduced to half in order to increase the yield. To this mix $0.43 \mu$ of $10 \%$ Tween-20 (P1379) and $3.33 \mu \mathrm{l}$ of $400 \mathrm{mM} \mathrm{NaHB3CN}$ (Sigma Aldrich 296945) was added, mixed gently by pipetting and incubated for $18-24 \mathrm{~h}$ at $37^{\circ} \mathrm{C}$ with mild agitation ( $\left.60 \mathrm{rpm}\right)$.

The next day $3.33 \mu \mathrm{l}$ of $65 \mathrm{mg} / \mathrm{ml}$ Carboxymethoxylamine (Sigma C13408) in $800 \mathrm{mM} \mathrm{NaOH}$ was added and incubated for $1 \mathrm{~h}$ at $37^{\circ} \mathrm{C}$ with mild agitation to stop the reaction. After incubation the tubes were centrifuged for $15 \mathrm{~min}$ at $16000 \mathrm{rpm}$ at $4^{\circ} \mathrm{C}$ and the supernatant discarded. The pellet was resuspended in $67 \mu \mathrm{l} 100 \mathrm{mM}$ Tris- $\mathrm{HCl}$ (pH 8.0), centrifuged again and was resuspended in $200 \mu$ l PBS with 0.05\% Proclin-300 (Sigma Aldrich 48912). The antibodies were vortexed briefly, spun down, and sonicated with 10 pulses before stored at $+4{ }^{\circ} \mathrm{C}$ until use.

AlphaLISA assay with conjugated beads. Samples were diluted in HBSS for optimal linearity for each antibody pair. For human samples, 300 cells/well were used for SOD- 1 and 3000 cells/well for $\beta$-tubulin. The PDX-samples were diluted $10 \mathrm{x}$ for tubulin and 500 times for SOD1. $3 \mu \mathrm{l}$ of each sample was loaded in AlphaPlate (Perkin Elmer 6008350), in duplicate when possible. $2 \mu \mathrm{l}$ biotinylated antibody was added to each well and the plate were then briefly spun down before addition of Acceptor beads. For both $\beta$-tubulin and SOD- 1 the biotinylated antibody (biotin-AF3418 and biotin-ab6046 respectively) were used at a final concentration of $3 \mathrm{nM}$.

Acceptor breads $(2 \mu \mathrm{l})$ were added and the plate was spun briefly and incubated on a shaker for $1 \mathrm{~h}$ at $60 \mathrm{rpm}$. For SOD-1, Acceptor beads were diluted to $45 \mu \mathrm{g} / \mathrm{ml}$ for a final concentration of $10 \mu \mathrm{g} / \mathrm{ml}$ and for $\beta$-tubulin T5201-conjugated Acceptor beads were used at a concentration of $180 \mu \mathrm{g}$ to get at final concentration of $40 \mu \mathrm{g} / \mathrm{ml}$. Then $2 \mu$ l Donor beads (Perkin Elmer 6760002S) was added at a concentration of $180 \mu \mathrm{g} / \mathrm{ml}$ for get at final concentration of $40 \mu \mathrm{g} / \mathrm{ml}$. Handling of beads was only done in a room with green light. Plates were sealed with sticky film, covered in foil, briefly spun down, and incubated in the dark over night before being read in an EnSpire 2300 (Perkin Elmer). 
ITDR-CETSA in multidrug-resistant K562 cells. For the intact cell ITDR-CETSA experiments, studied compounds (docetaxel, paclitaxel, or epothilone B) were prepared in RPMI supplemented with 5\% FBS at double the final compound concentration and with or without the addition of tariquidar (also at double the final concentration). Multidrug-resistant and parental K562 cells were harvested, washed in $+5 \%$ FBS, and resuspended at 6,66 million cells/ml in RPMI + 5\% FBS. Cells were then mixed 1:1 in PCR tubes with the previously prepared compound dilutions so that the final cell density was 3,33 million cells $/ \mathrm{ml}$ in $50 \mu$ lotal volume. The final tariquidar concentration in the allocated samples was $0,5 \mu \mathrm{M}$. Vehicle-treated samples were also included at a final concentration of $0,4 \%$ DMSO. The treated cells were incubated at $37^{\circ} \mathrm{C}$ for $1 \mathrm{~h} 45 \mathrm{~min}$ followed by heating for 3 minutes at $63^{\circ} \mathrm{C}$ in a Veriti thermal cycler (Applied Biosystems). Unheated samples were also included. Immediately after the heating, all samples were freeze-thawed three times using liquid nitrogen. The samples were vortexed after every freeze-thaw cycle. The remaining soluble $\beta$-tubulin was analyzed in the total lysate using AlphaLISA pair 8.

Resazurin cytotoxicity assay. For assessment of drug toxicity, multidrug-resistant and parental K562 cells in duplicates of 10000 cells/well, were seeded in black 96-well clear bottom polystyrene microplates (Sigma) containing various drug concentrations diluted in RPMI supplemented with $5 \%$ heat inactivated FBS. Following, $72 \mathrm{~h}$ incubation at $37^{\circ} \mathrm{C}, 10 \mu \mathrm{g} / \mathrm{ml}$ resazurin sodium salt (Sigma) was added to each well and further incubated for $2 \mathrm{~h}$ at $37^{\circ} \mathrm{C}$. Resorufin fluorescence was measured at a wavelength of $590 \mathrm{~nm}$ using an Enspire plate reader (Perkin Elmer).

Image-CETSA. Multidrug-resistant and parental K562 cells were propagated in RPMI-1640 medium supplemented with $10 \%$ heat inactivated FBS and continuously maintained at a cell density of $2 \times 10^{5}$ cells $/ \mathrm{ml}$. Drug resistance was preserved with $150 \mathrm{nM}$ vincristine or vehicle DMSO, in the resistant and parental K562 cells respectively, by selection for 72-96 h. Prior to each experiment, cells were washed once in HBSS before transfer to PCR tubes with RPMI-1640 medium containing 5\% heat inactivated FBS and various drug concentrations at a density of 4 million cells/ml. After $2 \mathrm{~h}$ incubation at $37^{\circ} \mathrm{C}$, CETSA heating was performed at $56^{\circ} \mathrm{C}$ in a Veriti Thermal Cycler (Applied Biosystems) for $3 \mathrm{~min}$. After heating, cells were collected by centrifugation at $200 \mathrm{rcf}$ for $3 \mathrm{~min}$ at $4{ }^{\circ} \mathrm{C}$ and washed twice in HBSS before transfer to black 96-well clear bottom polystyrene microplates (Sigma) pre-coated with $1 \%$ aqueous Alcian Blue solution (Electron Microscopy Sciences) for 15 min for cell adherence. Supernatant was aspirated after centrifugation at $200 \mathrm{rcf}$ for $5 \mathrm{~min}$ at $4{ }^{\circ} \mathrm{C}$, after which cells were fixed in $4 \% \mathrm{w} / \mathrm{v}$ formaldehyde (Thermo Scientific) for $15 \mathrm{~min}$ at room temperature. Next, the cells were washed twice in DPBS/Modified (GE Healthcare) and permeabilized by incubation with 5\% v/v Triton X-100 (Sigma) for 15 min at room temperature with gentle agitation. After an additional three washes, blocking was performed in $2 \% \mathrm{w} / \mathrm{v}$ bovine serum albumin (Sigma) for $1 \mathrm{~h}$ at room temperature with gentle agitation. $\beta$-tubulin conjugated Alexa Fluor 555 (\#3623, Cell Signaling) was diluted 1:200 in blocking buffer and added for overnight incubation protected from light at $4{ }^{\circ} \mathrm{C}$ with gentle agitation. Next, the cells were washed twice with $0.1 \% \mathrm{v} / \mathrm{v}$ Tween 20 (Sigma) and counterstained with $1 \mathrm{mg} / \mathrm{ml} \mathrm{v} / \mathrm{v}$ Hoechst (Chemometec) diluted in DPBS/Modified for $5 \mathrm{~min}$ at room temperature with gentle agitation. After an additional two washes, the Tween 20 wash buffer was replaced with DPBS/ Modified for subsequent image analysis.

Images and analysis were acquired with the Cytell ${ }^{\mathrm{TM}}$ Cell Imaging system (GE Healthcare) using a 2-colour BioApp specific for cytoplasmic image analysis. A blue (390/430) and a yellow (544/588) channel were applied for nuclear and cytoplasmic imaging respectively. Fifty-one fields covering the entire well were captured with a $10 \mathrm{x}$ Plan Apo 0.45 NA objective. Raw data based on average cellular cytoplasmic intensity for each individual well was further analyzed and plotted using GraphPad Prism version 6.

CETSA on in vivo treated xenografts. Female NOD-SCID mice were obtained from the breeding unit at the Department of Microbiology, Tumor and Cell Biology, Karolinska Institute. All mouse studies were approved by the Northern Stockholm Experimental Animal Ethical Committee (Dnr N 192/13 and Dnr N 2/17) and were performed in accordance with the relevant guidelines and regulations. Tumour volumes were measured with a caliper and calculated according to the standard formula (length $\times$ width $^{2} \times 0.52$ ).

For the in vivo experiments, NOD-SCID mice were injected with approximately $5 \times 10^{6} \mathrm{MCF}-7$ cells into a mammary gland. When the tumor sizes reached $0.2-0.3 \mathrm{~cm}^{3}$, the mice were injected with either vehicle (EtOH 1:1 in polysorbate 80 and then diluted in 5\% dextrose) or docetaxel 30 min before they were euthanized by inhalation of a lethal dose of $\mathrm{CO}_{2}$ followed by cervical dislocation. Tumours were removed and cut into pieces using a scalpel and put in PCR-strips (Applied Biosystems N8010580). $30 \mu \mathrm{l} \mathrm{HBSS} \mathrm{(Life} \mathrm{technologies}$ $\left.14025100+\mathrm{CaCl}_{2},+\mathrm{MgCl}_{2}\right)$ was added and the samples heated to different temperatures in a Veriti Thermal cycler (Applied Biosystems). After heating samples were frozen in liquid nitrogen and then thawed at $20^{\circ} \mathrm{C}$. High salt lysis buffer (50 mM tris- $\mathrm{HCl}, 300 \mathrm{mM} \mathrm{NaCl}, 0.5 \%$ NP40, $1 \mathrm{mM}$ EDTA, pH 7.2) and Halt Protease Inhibitor (Thermo Scientific 1861279) was added and the freeze-thawing repeated two additional times. The tumour pieces were further homogenized by crushing them with bent plastic pipette tips.

Soluble proteins were isolated by centrifugation at $20000 \mathrm{x}$ g for 20 minutes at $4{ }^{\circ} \mathrm{C}$ and the tubulin detected with western blot or AlphaLISA pair 2. SOD-1 was used as loading control in order to minimize the potential effect of differences in size of tumour pieces, this did however only give minimal change to the graph appearance, why we chose not to use this in the analysis of xenografts. Total five animals were use for the first pilot experiment and 16 mice were used for the second.

CETSA on ex vivo treated xenografts. NOD-SCID mice were injected with approximately 5 million MDA-MD-231 cells into a mammary gland. At the day of the experiment, four mice were euthanized by inhalation of a lethal dose of $\mathrm{CO}_{2}$ followed by cervical dislocation and tumours removed directly. Each tumour was 
divided into 12 pieces, which were incubated in vehicle (DMSO, 0.5\%) or $50 \mu \mathrm{M}$ docetaxel in HBSS (Life technologies $14025100+\mathrm{CaCl}_{2},+\mathrm{MgCl}_{2}$ ) supplemented with Halt Protease Inhibitor (Thermo Scientific 1861279) for $1 \mathrm{~h}$ at $37^{\circ} \mathrm{C}$. After incubation samples were heated to different temperatures in a Veriti Thermal cycler (Applied Biosystems). After heating samples were frozen in liquid nitrogen and then thawed at $20^{\circ} \mathrm{C}$. High salt lysis buffer $(50 \mathrm{mM}$ tris- $\mathrm{HCl}, 300 \mathrm{mM} \mathrm{NaCl}, 0.5 \% \mathrm{NP} 40,1 \mathrm{mM}$ EDTA, $\mathrm{pH}$ 7.2) was added and the freeze-thawing repeated two additional times. The tumour pieces were further homogenized by crushing them with bent plastic pipette tips. Soluble proteins were isolated by centrifugation at $20000 \mathrm{rcf}$ for 20 minutes at $4{ }^{\circ} \mathrm{C}$ and tubulin was detected with western blot.

CETSA on ex vivo treated PDX-tumours. These animal experiments were performed at the Erasmus University Medical Centre in Rotterdam and all experiments were approved by the Animal Experiment Committee under the Dutch Experiment on Animals Act and adhered to the European Convention for Protection of Vertebrate Animals used for Experimental Purposes (Directive 2010/63/EU).

Sixteen mice were inoculated with one of four prostate cancer cell lines bilaterally at 5 million cells on each side. The following prostate cancer cell lines were used: PC346C and PC339, and the docetaxel resistant lines PC346C-Doc and PC339-Doc. Cells were maintained as described previously ${ }^{37}$.

Tumour volume was monitored twice weekly by digital calipers, and tumours were excised 28-37 days after inoculation (tumour volumes ranged between 125 and $1500 \mathrm{~mm}^{3}$ ).

At the day of the experiment, mice were sacrificed (cervical dislocation), the tumours removed and fixed on a layer of low melting agarose using cyanoacrylate glue. The tumours were sliced using the Leica VT1200S Vibratome at a thickness of $300 \mu \mathrm{m}$ and the slices were transferred to PCR strips containing HBSS (Life technologies $14025100+\mathrm{CaCl}_{2},+\mathrm{MgCl}_{2}$ ) and treated with a range of drug concentrations as indicated for $1 \mathrm{~h}$ at $37^{\circ} \mathrm{C}$ and $5 \% \mathrm{CO}_{2}$. After incubation, samples were heated in a PCR-machine (Biometra T1 thermocycler) at $63^{\circ} \mathrm{C}$ for $3 \mathrm{~min}$ and then snap frozen in liquid nitrogen. All samples were stored at $-80^{\circ} \mathrm{C}$ until detection.

For detection, all samples were frozen and thawed three times in liquid nitrogen and a $20^{\circ} \mathrm{C}$ program on a PCR-machine. High salt lysis buffer $(50 \mathrm{mM}$ tris- $\mathrm{HCl}, 300 \mathrm{mM} \mathrm{NaCl}, 0.5 \% \mathrm{NP} 40,1 \mathrm{mM}$ EDTA, pH 7.2) was added and samples were vortexed vigorously before slices and debris were removed by centrifugation for $20 \mathrm{~min}$ at $1000 \mathrm{rpm}$. Supernatant was isolated and analyzed for $\beta$-tubulin and SOD- 1 content using AlphaLISA. For $\beta$-tubulin detection antibody pair 2 was used and the samples were diluted 10 times prior to AlphaLISA detection, while for SOD-1 samples were diluted 500 times.

Patient fine needle aspirates (FNAs). All experiments performed on human material were approved by the Regional Ethical Board in Stockholm. Samples were either anonymized (Dnr 2016 957-31) or taken from patients who signed informed consent (Dnr 2015/1694-31/1 with approved amendments 2016/2599-32, 2017/1353-32 and 2017/1267-32). All methods were performed in accordance with the relevant guidelines and regulations. FNAs were taken by pathologists or cytologists from tumours after surgery or directly from patients and placed in tubes with L15 media (Gibco L-15 21083-027). Cells were counted, spun down, and resuspended in HBSS (Life technologies $14025100+\mathrm{CaCl}_{2},+\mathrm{MgCl}_{2}$ ) and treated with docetaxel for $15 \mathrm{~min}$. When 100000 cells or more were obtained, cells were resuspended to a final concentration of 3 million cells $/ \mathrm{ml}$ and detected with AlphaLISA pair 8 . When 20000 to 10000 cells were obtained cells were suspended to $1 \mathrm{million} / \mathrm{ml}$ and detected with conjugated AlphaLISA pair 8.

Data analysis. The presented data were generated in independent experiments $\mathrm{n} \geq 3$ except Figs. 2C-E, S2D-F and Fig. 7, where data correspond to technical duplicates. All graphs were generated using GraphPad Prism (versions 6-8). All data are presented as mean with error bars representing the standard error of the mean (S.E.M). Error bars that are smaller than the displayed data points are not shown by the software e.g. in Fig. 2C.

ITDR-CETSA and cell viability data are presented as a percentage of the signal corresponding to the compound concentration where maximum stabilization was achieved in each series, with the exception of Figs. $6 \mathrm{E}$ and S5E where data are presented as normalized to SOD-1, Fig. 6B-D where data are presented as a percentage of the signal obtained for the drug-sensitive cells at maximum stabilization, and Fig. 7B,C where data are presented as a percentage of the signal detected for the untreated samples in each series. Sigmoidal curves were fit in GraphPad Prism using non-linear regression of the type [Inhibitor] vs. Response (three parameters) with the function $\mathrm{Y}=$ Bottom $+($ Top-Bottom $) /(1+(\mathrm{X} / \mathrm{IC50}))$. ITDR-CETSA curves in Fig. 7 were fit using non-linear regression of the type [Inhibitor] vs. Response (four parameters) with the function $\mathrm{Y}=\mathrm{Bottom}+($ Top-Bottom)/ $\left(1+(\mathrm{IC} 50 / \mathrm{X})^{\wedge}\right.$ HillSlope $)$. The $\mathrm{EC}_{50}$ values with associated standard errors and $95 \%$ confidence intervals calculated for the curves are presented in corresponding tables. The statistical significance of the difference observed between the dose-response curves in each graph was calculated using a T-test for the $\mathrm{EC}_{50}$ values obtained in each individual experiment.

For CETSA melt curves (Figs. 1B-G, 2C,D, 4A and 5A-C), data are presented as a percentage of the signal detected at the lowest temperature in each melt curve and a line connecting the data points was automatically generated by the abovementioned software. Melting temperatures (Tm) and the size of the CETSA shifts $(\Delta \mathrm{Tm})$ were also determined in GraphPad Prism. One-way ANOVA was used for analyzing the CETSA melt curve data in Figs. 1B-G and 5A-C. The significance levels for these statistical analyses are presented in corresponding tables.

\section{Data availability}

The datasets generated and analysed during the current study are available from the corresponding author on reasonable request. 
Received: 17 June 2019; Accepted: 27 November 2019;

Published online: 18 December 2019

\section{References}

1. Dumontet, C. \& Jordan, M. A. Microtubule-binding agents: a dynamic field of cancer therapeutics. Nat Rev Drug Discov 9, 790-803, https://doi.org/10.1038/nrd3253 (2010).

2. Ercolak, V., Sahin, B., Gunaldi, M., Duman, B. B. \& Afsar, C. U. Efficacy of paclitaxel in the treatment of Kaposi sarcoma. Eur Rev Med Pharmacol Sci 19, 4095-4100 (2015).

3. Prithviraj, G. K. et al. Carboplatin and paclitaxel as first-line treatment of unresectable or metastatic esophageal or gastric cancer. Dis Esophagus 28, 782-787, https://doi.org/10.1111/dote.12279 (2015).

4. Perez, E. A. Paclitaxel in Breast Cancer. Oncologist 3, 373-389 (1998)

5. King, K. M. et al. Optimal use of taxanes in metastatic breast cancer. Curr Oncol 16, 8-20 (2009).

6. Tannock, I. F. et al. Docetaxel plus prednisone or mitoxantrone plus prednisone for advanced prostate cancer. N Engl J Med 351, 1502-1512, https://doi.org/10.1056/NEJMoa040720 (2004).

7. de Bono, J. S. et al. Prednisone plus cabazitaxel or mitoxantrone for metastatic castration-resistant prostate cancer progressing after docetaxel treatment: a randomised open-label trial. Lancet 376, 1147-1154, https://doi.org/10.1016/S0140-6736(10)61389-X (2010).

8. Sathianathen, N. J. et al. Taxane-based chemohormonal therapy for metastatic hormone-sensitive prostate cancer. Cochrane Database Syst Rev 10, CD012816, https://doi.org/10.1002/14651858.CD012816.pub2 (2018).

9. Field, J. J., Díaz, J. F. \& Miller, J. H. The binding sites of microtubule-stabilizing agents. Chem Biol 20, 301-315, https://doi. org/10.1016/j.chembiol.2013.01.014 (2013).

10. Dorr, R. T. Pharmacology of the taxanes. Pharmacotherapy 17, 96S-104S (1997).

11. Trock, B. J., Leonessa, F. \& Clarke, R. Multidrug resistance in breast cancer: a meta-analysis of MDR1/gp170 expression and its possible functional significance. I Natl Cancer Inst 89, 917-931 (1997).

12. Orr, G. A., Verdier-Pinard, P., McDaid, H. \& Horwitz, S. B. Mechanisms of Taxol resistance related to microtubules. Oncogene 22, 7280-7295, https://doi.org/10.1038/si.onc.1206934 (2003).

13. Murray, S., Briasoulis, E., Linardou, H., Bafaloukos, D. \& Papadimitriou, C. Taxane resistance in breast cancer: mechanisms, predictive biomarkers and circumvention strategies. Cancer Treat Rev 38, 890-903, https://doi.org/10.1016/j.ctrv.2012.02.011 (2012).

14. Parker, A. L., Teo, W. S., McCarroll, J. A. \& Kavallaris, M. An Emerging Role for Tubulin Isotypes in Modulating Cancer Biology and Chemotherapy Resistance. Int J Mol Sci 18, https://doi.org/10.3390/ijms18071434 (2017).

15. Ferlini, C. et al. Bcl-2 down-regulation is a novel mechanism of paclitaxel resistance. Mol Pharmacol 64, 51-58, https://doi. org $/ 10.1124 / \mathrm{mol} .64 .1 .51(2003)$

16. Wang, Z., Goulet, R., Stanton, K. J., Sadaria, M. \& Nakshatri, H. Differential effect of anti-apoptotic genes Bcl-xL and c-FLIP on sensitivity of MCF-7 breast cancer cells to paclitaxel and docetaxel. Anticancer Res 25, 2367-2379 (2005).

17. Mabuchi, S. et al. Inhibition of inhibitor of nuclear factor-kappaB phosphorylation increases the efficacy of paclitaxel in in vitro and in vivo ovarian cancer models. Clin Cancer Res 10, 7645-7654, https://doi.org/10.1158/1078-0432.CCR-04-0958 (2004).

18. Gerritsen-van Schieveen, P., Royer, B. \& Therapeutics, T. d. m. g. o. t. F. S. o. P.a. Level of evidence for therapeutic drug monitoring of taxanes. Fundam Clin Pharmacol 25, 414-424, https://doi.org/10.1111/j.1472-8206.2010.00874.x (2011)

19. Martinez Molina, D. et al. Monitoring drug target engagement in cells and tissues using the cellular thermal shift assay. Science 341, 84-87, https://doi.org/10.1126/science.1233606 (2013).

20. Ishii, T. et al. CETSA quantitatively verifies in vivo target engagement of novel RIPK1 inhibitors in various biospecimens. Sci Rep 7, 13000, https://doi.org/10.1038/s41598-017-12513-1 (2017).

21. Savitski, M. M. et al. Tracking cancer drugs in living cells by thermal profiling of the proteome. Science 346, 1255784, https://doi. org/10.1126/science.1255784 (2014)

22. Jordan, M. A. \& Wilson, L. Microtubules as a target for anticancer drugs. Nat Rev Cancer 4, 253-265, https://doi.org/10.1038/ $\operatorname{nrc1317}(2004)$

23. Downing, K. H. Structural basis for the interaction of tubulin with proteins and drugs that affect microtubule dynamics. Annu Rev Cell Dev Biol 16, 89-111, https://doi.org/10.1146/annurev.cellbio.16.1.89 (2000).

24. Vindya, N. G., Sharma, N., Yadav, M. \& Ethiraj, K. R. Tubulins - the target for anticancer therapy. Curr Top Med Chem 15, 73-82 (2015).

25. Tan, C. S. H. et al. Thermal proximity coaggregation for system-wide profiling of protein complex dynamics in cells. Science 359, 1170-1177, https://doi.org/10.1126/science.aan0346 (2018).

26. Dai, L. et al. Horizontal Cell Biology: Monitoring Global Changes of Protein Interaction States with the Proteome-Wide Cellular Thermal Shift Assay (CETSA). Annu Rev Biochem. https://doi.org/10.1146/annurev-biochem-062917-012837 (2019).

27. Miettinen, T. P. \& Björklund, M. NQO2 is a reactive oxygen species generating off-target for acetaminophen. Mol Pharm 11, 4395-4404, https://doi.org/10.1021/mp5004866 (2014).

28. Löfgren, C. et al. Mechanisms of cross-resistance between nucleoside analogues and vincristine or daunorubicin in leukemic cells. Biochem Biophys Res Commun 320, 825-832, https://doi.org/10.1016/j.bbrc.2004.06.016 (2004).

29. Axelsson, H. et al. In Situ Target Engagement Studies in Adherent Cells. ACS Chem Biol 13, 942-950, https://doi.org/10.1021/ acschembio.7b01079 (2018).

30. Massey, A. J. A high content, high throughput cellular thermal stability assay for measuring drug-target engagement in living cells. PLoS One 13, e0195050, https://doi.org/10.1371/journal.pone.0195050 (2018).

31. Nair, A. B. \& Jacob, S. A simple practice guide for dose conversion between animals and human. J Basic Clin Pharm 7, 27-31, https:// doi.org/10.4103/0976-0105.177703 (2016).

32. de Morrée, E. et al. Understanding taxanes in prostate cancer; importance of intratumoral drug accumulation. Prostate 76, 927-936, https://doi.org/10.1002/pros.23182 (2016).

33. Azarenko, O., Smiyun, G., Mah, J., Wilson, L. \& Jordan, M. A. Antiproliferative mechanism of action of the novel taxane cabazitaxel as compared with the parent compound docetaxel in MCF7 breast cancer cells. Mol Cancer Ther 13, 2092-2103, https://doi. org/10.1158/1535-7163.MCT-14-0265 (2014).

34. de Morrée, E. S. et al. Loss of SLCO1B3 drives taxane resistance in prostate cancer. Br J Cancer 115, 674-681, https://doi.org/10.1038/ bjc.2016.251 (2016).

35. Jarvis, C., Nelius, T., Martinez-Marin, D., Sennoune, S. R. \& Filleur, S. Cabazitaxel regimens inhibit the growth of prostate cancer cells and enhances the anti-tumor properties of PEDF with various efficacy and toxicity. Prostate 78, 905-914, https://doi. org/10.1002/pros.23647 (2018).

36. Ernst, L. M. \& Rimm, D. L. Quantitative examination of mechanophysical tumor cell enrichment in fine-needle aspiration specimens. Cancer 96, 275-279, https://doi.org/10.1002/cncr.10746 (2002).

37. Marques, R. B. et al. The human PC346 xenograft and cell line panel: a model system for prostate cancer progression. Eur Urol 49, 245-257, https://doi.org/10.1016/j.eururo.2005.12.035 (2006). 


\section{Acknowledgements}

We would like to acknowledge Sören Lehmann and Christer Paul at the Karolinska Institute for kindly providing us with the multidrug-resistant K562 cells, Eva Darai Ramkvist, Edneia Tani and the puncture reception at Radiumhemmet for collecting the pre-operative biopsies, and Johanna Klinge, Helen Eriksson, Lisa Viberg, Susanne Agartz, Ran Ma, and Xinsong Chen for help with administration and logistics of clinical samples. PN acknowledges grants from the Swedish Research Council (VR-MH and VR-NT), the Swedish Cancer Society, Radiumhemmet's funds, the Swedish Childhood Cancer Fund, and the Knut and Alice Wallenberg Foundation. JB acknowledges grants from PCM/Radiumhemmet's funds, and the Knut and Alice Wallenberg Foundation. Open access funding provided by Karolinska Institute.

\section{Author contributions}

Designed study: P.N., S.L., W.v.W., J.B., Y.C., J.H. Performed experiments: A.L., S.B., H.L., L.M., S.E.S., A.R., S.L., T.S. Patient samples logistics: J.H., A.B., S.L., A.L., S.B., H.L., A.R. Drafted manuscripts: A.L., P.N., S.L. Final comments on manuscript: All.

\section{Competing interests}

$\mathrm{PN}$ is the inventor of CETSA, holder of patents related to the CETSA method, and the co-founder of Pelago Bioscience AB. All the other authors declare no competing interests.

\section{Additional information}

Supplementary information is available for this paper at https://doi.org/10.1038/s41598-019-55526-8.

Correspondence and requests for materials should be addressed to P.N.

Reprints and permissions information is available at www.nature.com/reprints.

Publisher's note Springer Nature remains neutral with regard to jurisdictional claims in published maps and institutional affiliations.

(c) (i) Open Access This article is licensed under a Creative Commons Attribution 4.0 International

License, which permits use, sharing, adaptation, distribution and reproduction in any medium or format, as long as you give appropriate credit to the original author(s) and the source, provide a link to the Creative Commons license, and indicate if changes were made. The images or other third party material in this article are included in the article's Creative Commons license, unless indicated otherwise in a credit line to the material. If material is not included in the article's Creative Commons license and your intended use is not permitted by statutory regulation or exceeds the permitted use, you will need to obtain permission directly from the copyright holder. To view a copy of this license, visit http://creativecommons.org/licenses/by/4.0/.

(c) The Author(s) 2019 